\title{
Importin- $\beta 11$ Regulates Synaptic Phosphorylated Mothers Against Decapentaplegic, and Thereby Influences Synaptic Development and Function at the Drosophila Neuromuscular Junction
}

\author{
Misao E. Higashi-Kovtun, ${ }^{1,2}$ Timothy J. Mosca, ${ }^{1,2}$ Dion K. Dickman, ${ }^{1,2}$ Ian A. Meinertzhagen, ${ }^{3}$ and Thomas L. Schwarz ${ }^{1,2}$ \\ ${ }^{1}$ F. M. Kirby Center for Neurobiology, Children's Hospital, Boston, Massachusetts 02115, ${ }^{2}$ Department of Neurobiology, Harvard Medical School, Boston, \\ Massachusetts 02115, and ${ }^{3}$ Department of Psychology, Life Sciences Centre, Dalhousie University, Halifax, Nova Scotia B3H 4J1, Canada
}

\begin{abstract}
Importin proteins act both at the nuclear pore to promote substrate entry and in the cytosol during signal trafficking. Here, we describe mutations in the Drosophila gene importin- $\boldsymbol{\beta} 11$, which has not previously been analyzed genetically. Mutants of importin- $\boldsymbol{\beta} 11$ died as late pupae from neuronal defects, and neuronal importin- $\beta 11$ was present not only at nuclear pores but also in the cytosol and at synapses. Neurons lacking importin- $\beta 11$ were viable and properly differentiated but exhibited discrete defects. Synaptic transmission was defective in adult photoreceptors and at larval neuromuscular junctions (NMJs). Mutant photoreceptor axons formed grossly normal projections and synaptic terminals in the brain, but synaptic arbors on larval muscles were smaller while still containing appropriate synaptic components. Bone morphogenic protein (BMP) signaling was the apparent cause of the observed NMJ defects. Importin- $\boldsymbol{\beta} 11$ interacted genetically with the BMP pathway, and at mutant synaptic boutons, a key component of this pathway, phosphorylated mothers against decapentaplegic (pMAD), was reduced. Neuronal expression of an importin- $\boldsymbol{\beta} 11$ transgene rescued this phenotype as well as the other observed neuromuscular phenotypes. Despite the loss of synaptic pMAD, pMAD persisted in motor neuron nuclei, suggesting a specific impairment in the local function of pMAD. Restoring levels of pMAD to mutant terminals via expression of constitutively active type I BMP receptors or by reducing retrograde transport in motor neurons also restored synaptic strength and morphology. Thus, importin- $\beta 11$ function interacts with the BMP pathway to regulate a pool of pMAD that must be present at the presynapse for its proper development and function.
\end{abstract}

\section{Introduction}

The complex and dynamic nature of neuronal circuits necessitates well-orchestrated signals to direct the development, maintenance, and plasticity of synapses. These signals include both local responses to a synapse's milieu and nuclear alterations in gene transcription, and neurons therefore require that the nu-

Received July 31, 2009; revised Feb. 11, 2010; accepted March 1, 2010.

This work was supported by National Institutes of Health (NIH) Grants R01 NS041062 and MH075058 (T.L.S.) and EY03592 (I.A.M.), and predoctoral fellowships from NIH/National Institute of Neurological Disorders and Stroke Ruth L. Kirschstein National Research Service Award (M.E.H.K.), the National Defense Science and Engineering Graduate Fellowship (T.J.M.), and the Howard Hughes Medical Institute (D.K.D.). We thank D. Schmucker for assistance in generating transgenic flies, D. Allan for intellectual input and Drosophila stocks, T. Herman for R7 photoreceptor projection analysis, A. Deb and E. Pogoda for technical assistance, and L. Bu of the Mental Retardation Developmental Disabilities Research Center Imaging Core under the support of National Institute of Child Health and Human Development Grant P3OHD18655 for imaging assistance. We thank A. DiAntonio, N. Reist, P. ten Dijke, M. Noll, W. Boll, and M. Gonzales-Gaitan for antibodies and Drosophila stocks. We also thank the Bloomington Stock Center at the University of Indiana for fly stocks and the Developmental Studies Hybridoma Bank at the University of lowa for antibodies.

Correspondence should be addressed to Thomas L. Schwarz, F. M. Kirby Center for Neurobiology, Children's Hospital, 300 Longwood Avenue, CLS 12120, Boston, MA 02115. E-mail: thomas.schwarz@childrens.harvard.edu.

D. K. Dickman's present address: Department of Biochemistry and Biophysics, University of California, San Francisco, 1550 Fourth Street, San Francisco, CA 94158.

D0I:10.1523/JNEUROSCI.3739-09.2010

Copyright $\odot 2010$ the authors $\quad 0270-6474 / 10 / 305253-16 \$ 15.00 / 0$ cleus receive and interpret signals from the cells periphery (Goodman and Shatz, 1993; Greer and Greenberg, 2008).

One potential nexus for the interaction of local and nuclear signaling is the importin protein family. These proteins have a canonical function as nuclear import receptors that regulate transport from the cytoplasm to the nucleus, thereby fulfilling the need of proteins larger than $30-60 \mathrm{kDa}$ to transit the nuclear pore in an actively catalyzed manner (Paine et al., 1975; Peters, 1983; Görlich and Kutay, 1999). Because nuclear entry can be a carefully regulated process for many signaling molecules that govern transcription, importins are critical to regulating neuronal properties (Otis et al., 2006; Ting et al., 2007; Perry and Fainzilber, 2009).

There are over 20 importin genes in mammals. This diversity permits the specialization of importins for particular cargo classes and the independent and tissue-specific regulation of their nuclear import (Fried and Kutay, 2003). In the classical nuclear import pathway, a cargo containing a nuclear localization signal binds to an importin- $\alpha$, which in turn binds to an importin- $\beta$. The latter, via its interactions with nucleoporins, shuttles the heteromer-cargo complex into the nucleus (Görlich et al., 1995; Moroianu et al., 1995). Importin- $\beta$ s however, can also promote nuclear entry by direct interaction with cargo independent of an 
importin- $\alpha$ (Görlich and Kutay, 1999). Directionality is tightly regulated by the small GTPase Ran, which is GTP-bound in the nucleus, where it dissociates importins from their cargo (Rexach and Blobel, 1995; Görlich and Kutay, 1999).

Importins, however, are not spatially restricted to the nucleus and can occupy roles beyond that of nuclear gatekeeper (Jakel et al., 2002; Vrailas et al., 2006; James et al., 2007). In the nervous system, particularly, they have gained significant attention for their role in synapse targeting (Ting et al., 2007) and signaling from the periphery to the nucleus (Otis et al., 2006; Perry and Fainzilber, 2009). Axonal injury causes the local translation of importin- $\beta$, which then translocates with its cargo to the soma to promote neurite re-outgrowth (Hanz et al., 2003; Perlson et al., 2005). Similarly, synaptic stimuli cause importins to move from dendrites to nuclei to effect forms of synaptic plasticity (Thompson et al., 2004; Lai et al., 2008). Importins are thus emerging as fundamental regulators of neuronal function (Otis et al., 2006; Perry and Fainzilber, 2009).

In a forward genetic screen for defective synaptic transmission, mutants were isolated in Drosophila importin- $\beta 11$ (imp $\beta 11$ ), also called ran-binding protein 11, one of 12 importins identified in the Drosophila genome. In mammalian cells, importin- $\beta 11$ is required for nuclear import of the ubiquitin conjugating enzyme UbcM2 (Plafker and Macara, 2000; Plafker et al., 2004) and the ribosomal protein rpL12 (Plafker and Macara, 2002), but no neuronal functions are known. Because only one importin- $\beta$ has been characterized at the Drosophila larval neuromuscular junction (NMJ), importin- $\beta 13$ (Giagtzoglou et al., 2009), we undertook to characterize the imp $\beta 11$ mutants. We found that loss of imp $\beta 11$ caused a local misregulation of bone morphogenic protein (BMP) signaling at the larval NMJ, which resulted in a reduction in both the number of boutons and synaptic transmission.

\section{Materials and Methods}

Fly stocks. Imp $\beta 11$ alleles were isolated as described previously (Dickman et al., 2005). The $y, w$;FRT42D GMR-hid $y+c l$ 2R/CyO;EGUF/EGUF parental stock used in the mutagenesis was described previously (Stowers and Schwarz, 1999).

Five independent alleles of $i m p \beta 11$ were isolated and mapped using the deficiencies $D f(2 R) \Delta M 22-103$ (Poxn ${ }^{\Delta \text { M22-103 }}$ ), a generous gift from M. Noll and W. Boll (University of Zurich, Zurich, Switzerland) (Boll and Noll, 2002), $D f(2 R) J p 1$, and $D f(2 R) J p 5$ (Bloomington Stock Center) followed by genomic sequencing to identify the precise molecular lesions. For all experiments, the control genotype was $y, w$; FRT42D (Xu and Rubin, 1993), whereas for imp $\beta 11$ mutants, the $i m p \beta 11^{70}$ allele was used in trans to the genomic deficiency $D f(2 R) \Delta M 22-103$.

$U A S$-imp $\beta 11-e G F P$ was generated by cloning the full-length cDNA (EST, LD41918; Invitrogen) into the $p U A S T$ vector (Brand and Perrimon, 1993) using PCR-added BglII and KpnI sites. Enhanced green fluorescent protein (eGFP) was ligated in frame into the $p U A S T$-imp $\beta 11$ construct using engineered $K p n \mathrm{I}$ and $\mathrm{Xba \textrm {I }}$ sites. Transgenic flies were generated by standard transformation methods (Rubin and Spradling, 1982).

All fly stocks were raised in humidified incubators at $25^{\circ} \mathrm{C}$. Thirdinstar larvae were grown in low-density cages at $25^{\circ} \mathrm{C}$ on agar grape plates with yeast paste.

The following stocks were also used: elav-Gal4 (Luo et al., 1994); daGal4 (Wodarz et al., 1995); G14-Gal4, OK6-Gal4, wit ${ }^{A 12}$, wit ${ }^{B 11}$, wit ${ }^{H A 1}$ (Aberle et al., 2002); $n$-syb ${ }^{F-33 R}$ (Deitcher et al., 1998); MAD ${ }^{10}$ (Sekelsky et al., 1995); UAS-gbb ${ }^{9.1}$ (S. Thor, Linköping University, Linköping, Sweden); (Wharton et al., 1999); UAS-tkv ${ }^{\text {Q253D }}$ (mobilized onto X by D. Allan, University of British Columbia, Vancouver, BC Canada) (Nellen et al., 1996); UAS-sax Q263D (mobilized onto X by D. Allan) (Haerry et al., 1998); UAS-GFP-MAD (Dudu et al., 2006); UAS-dynamitin (D. Allan) (Duncan and Warrior, 2002); tubulin-GAL4 (Lee and Luo, 1999); UAS- wit (Marqués et al., 2002). Stocks were obtained from the Bloomington Stock Center when available.

Antibody production. A PCR fragment with flanking 5' EcoRI and 3' AvaI sites encoding importin- $\beta 11$ amino acids 923 to 1075 was amplified and cloned into the pGEX-4T-1 vector (GE Healthcare) using the following primers: 5' GST-RanB2- GAA TTC GGC GAA GTG ATG GAC AA 3' GST-RanB2-GAG CTC CGG CCT GAG GTG GAC AA. The fidelity of the clone was verified by sequencing throughout the entire open reading frame. Subsequently, the $51 \mathrm{kDa}$ glutathione $S$-transferase (GST) fusion protein was expressed in and purified from Escherichia coli BL21 cells using the Bulk GST Fusion Purification Module according to the manufacturer's protocols (GE Healthcare). The resultant protein was injected into naive New Zealand white rabbits (Covance). Crude antisera were then affinity purified over an Affi-Prep 10 column (Bio-Rad) containing the original fusion protein to which the antibody was raised. Affinitypurified antibody was eluted from the column by standard methods (Harlow and Lane, 1988) in $100 \mathrm{~mm}$ glycine, $\mathrm{pH}$ 2.3, and neutralized in 1 $\mathrm{M}$ Tris base, $\mathrm{pH}$ 7.5. The antibodies were then dialyzed with PBS containing $5 \%$ BSA. Antibodies were tested by recognition of a $110 \mathrm{kDa}$ band by Western blot corresponding to the predicted molecular weight of importin- $\beta 11$ that was absent in null mutants. One antibody, 5157 , was found to recognize endogenous and exogenous importin- $\beta 11$ and was used for subsequent studies.

Germline clones. The following stocks and genotypes were used: (1) $y, w, h s-F L P ; F R T 42 D$ imp- $\beta 11^{70} / C y O$; (2) $y, w, h s-F L P$; FRT42D; (3) $y, w$; FRT42D imp- $\beta 11^{70} / C y o$, GFP [CyO, P(ActGFP)]; (4) $y, w$; FRT42D; (5) $\left.w{ }^{*}\right] ; P\{w[+m W . h s]=F R T(w[h s])\} G 13 P\{w[+m C]=$ ovoD1-18 $\} 2 R /$ $D p(? ; 2) b w[D], S[1] w g[S p-1] M s(2) M[1] b w[D] / C y O ;+;+(B L 2125)$; (6) $y, w, h s-F L P / y, w$; FRT42B ovo $/ F R T 42 D$ imp- $\beta 11^{70}$; (7) $y, w$, hs-FLP/ $y, w ;$ FRT42B ovo $D / F R T 42 D$.

Germline clones were generated using the dominant female sterile technique using the ovo ${ }^{D}$ mutation and a source of FLPase under the control of a heat-shock promoter (Chou and Perrimon, 1992, 1996). In experimental conditions, FRT42B ovo ${ }^{D}$-bearing males were crossed to $y, w, h s-F L P ; F R T 42 D i m p \beta 11^{70} / C y O$ virgins. The progeny were heat shocked at $37^{\circ} \mathrm{C}$ starting in the second instar for $1 \mathrm{~h}$ per day for 6 consecutive days to induce recombination. Though the recombination of $42 \mathrm{~B}$ and $42 \mathrm{D}$ is expected to cause a chromosomal deletion between these two loci, this deletion has been shown previously to have no impact on the production of viable embryos (Murthy et al., 2005). Indeed, control crosses using the parental 42D chromosome resulted in embryos that developed into normal adults. Resultant virgin female adults of the genotype $y, w, h s-F L P / y, w ; F R T 42 B$, ovo ${ }^{D} / F R T 42 D$, imp $\beta 11^{70}$ were then mated to $y, w$; FRT $42 D$, imp $\beta 11^{70} / C y o$, GFP [CyO, P\{ActGFP $\left.\}\right]$ with the intent to select against GFP-bearing balancer chromosomes to ensure both a germline null and a zygotic null. Results were confirmed independently using the imp $\beta 11^{20}$ allele.

Immunostaining. Third-instar larval fillets were fixed in $4 \%$ paraformaldehyde for $20 \mathrm{~min}$. Antibody staining was performed in PBS containing $0.3 \%$ Triton X-100 and 5\% normal donkey serum. Fillets were mounted in Vectashield (Vector Labs). Adult recombinant heads were prepared as described previously (Stowers et al., 2002) with the exception of fixation conditions (3.7\% formaldehyde in 100 mu potassium phosphate, $\mathrm{pH} 6.8,450 \mathrm{~mm} \mathrm{KCl}, 150 \mathrm{~mm} \mathrm{NaCl}$, and 20 $\mathrm{mm} \mathrm{MgCl}^{2}$ ) and sectioned using a Leica CM3050S cryostat. For comparisons, animals were stained concurrently, imaged, and processed identically.

The following antibodies were used: anti-Chaoptin 24B10 [1:100; Developmental Studies Hybridoma Bank (DSHB)]; rabbit anti-synaptotagmin I (Syt I; 1:4000) (Mackler et al., 2002); mouse anti-Bruchpilot (BRP), nc82 (1:100) (Hofbauer et al., 2009); mouse anti-GluRIIA (1:100; DSHB); rabbit anti-GluRIIB, (1:2500) (Marrus et al., 2004); rabbit anti-GluRIIC (1:2000) (Marrus and DiAntonio, 2004); rabbit anti-phosphorylated mothers against decapentaplegic (pMAD), PS1 (1:2000) (Persson et al., 1998); mouse anti-Fasciclin II (FasII), 1D4 (1:20; DSHB); mouse antiFutsch, 22C10 (1:100; DSHB); rabbit anti-Thickveins (Tkv) (1:1250) (McCabe et al., 2004); mouse anti-Wishful thinking (Wit), 23C7 (1:10) (Aberle et al., 2002; Wang et al., 2007); goat anti-MAD (1:100; Santa Cruz Biotechnology) (Zhu et al., 1999; Li and Li, 2006); cyanine 5 
(Cy5)- or FITC-conjugated goat anti-HRP (1:100; Jackson ImmunoResearch); Alexa Fluor 594-conjugated wheat germ agglutinin at 5 $\mu \mathrm{M}$ (Invitrogen); FITC- or Cy3-conjugated secondary antibodies (1: 200; Jackson ImmunoResearch).

Imaging and analysis parameters. All images were processed using a Zeiss LSM 510 Meta laser scanning confocal microscope and either a $63 \times, 1.4$ numerical aperture (NA) or $40 \times, 1.0$ NA oil-immersion objective. Laser scanning microscope software and Adobe Photoshop (Adobe Systems) were used for image processing. For bouton quantification, larvae were stained with antibodies to HRP and boutons counted on muscles 6 and 7 in segment A2. Muscle surface area was calculated from $10 \times$ differential interference contrast micrographs of muscle fields; the length and width of each muscle were used to calculate a surface area. Statistics were performed as repeated-measurement ANOVA tests with a post hoc Dunnett's multiple comparison test between all combinations of genotypes. BRP, GluRIIA, GluRIIB, and GluRIIC puncta were manually counted at MN4b synapses on muscle 4 . Terminal area was calculated using the threshold function in MetaMorph for the HRP channel. Statistical analysis was conducted using GraphPad Prism 5 software and significance values were calculated using an unpaired Student's $t$ test. All histograms and measurements are shown as mean \pm SEM; sample size $(n)$ is described either in the figure legends or in the Results section.

Electrophysiology. NMJ recordings were performed as described previously (Dickman et al., 2005). Briefly, recordings were conducted in HL3 saline solution (Stewart et al., 1994) containing $20 \mathrm{~mm} \mathrm{Mg}^{2+}$ and either $1 \mathrm{mM} \mathrm{Ca}^{2+}$ or $0.6 \mathrm{~mm} \mathrm{Ca}^{2+}$ as indicated. All physiology was recorded from muscle 6 in abdominal segments A2 to A4. Recordings conducted on muscles with membrane potentials $\left(V_{\mathrm{m}}\right)$ of $-55 \mathrm{mV}$ or less were included for analysis. Quantal content was corrected for nonlinear summation (Martin, 1955).

Electroretinograms (ERGs) were performed as described previously (Dickman et al., 2005). HL3 saline solution was used in the stimulating electrode placed in the thorax of the adult fly. The recording electrode was filled with $3 \mathrm{M} \mathrm{KCl}$ and placed on the surface on the eye. Light stimuli lasted $1 \mathrm{~s}$.

Philanthotoxin (PhTx) homeostasis experiments were conducted as described previously (Goold and Davis, 2007). A final concentration of 4 $\mu \mathrm{M}$ PhTx in $1 \mathrm{mM} \mathrm{Ca}^{2+}$ HL3 saline was applied to a semi-intact larval preparation for $10 \mathrm{~min}$. The PhTx was washed out, and recordings were made as above.

Statistics were calculated using ANOVA followed by a Bonferroni post-comparison test (GraphPad). All histograms and measurements are shown as mean \pm SEM; sample size $(n)$ is described either in the figure legends or in the Results section.

Electron microscopy. Laminas innervated by either control or imp $\beta 11$ mutant photoreceptors were prepared for electron microscopy (EM) using previously reported methods (Meinertzhagen, 1996). Single sections containing cartridge profiles cut in fair cross-section were examined, and digital montages were collected from images obtained with a Philips Tecnai 12 electron microscope operated at $80 \mathrm{kV}$, using a Kodak Megaview II camera with software (AnalySIS; Soft Imaging System).

We made counts of the following organelle profiles: $\sim 30 \mathrm{~nm}$ synaptic vesicles, T-bar presynaptic ribbons at tetrad synapses, and glial invaginations into R1-R6 terminals, called capitate projections. The perimeters and cross-sectional areas of terminal profiles were measured with software (NIH ImageJ) to determine the packing density of synaptic vesicle profiles. Tests for statistical significance in organelle counts were first made by an unweighted means ANOVA, followed by Tukey's honestly significant difference test using software (Systat version 5.2.1). Error bars in figures indicate mean \pm SEM.

Immunoblots and SDS-PAGE analysis. For immunoblots, extracts were made from timed embryo collections or from intact third-instar larvae by drop-freezing specimens in liquid $\mathrm{N}_{2}$ and homogenizing them directly in $2 \times$ Laemmli buffer (Laemmli, 1970). Synaptosome fractions were prepared from adult heads as described previously (Kelly, 1983) in synaptosome buffer (10 mM HEPES, pH 7.5, $1 \mathrm{~mm} \mathrm{MgCl}_{2}, 0.32 \mathrm{M}$ sucrose). Proteins were separated on $8 \%$ polyacrylamide gels and transferred to nitrocellulose membranes. Primary antibodies were applied overnight at $4^{\circ} \mathrm{C}$, and secondary antibodies for $1 \mathrm{~h}$ at $21^{\circ} \mathrm{C}$. The following antibodies were used: rabbit anti-importin- $\beta 11$ (1:7500; present study); mouse anti-Bruchpilot (1:100) (Hofbauer et al., 2009); mouse anti-Lamin C LC28.26 and anti-Lamin $\mathrm{D}_{\mathrm{m}} 0$ ADL101 (1:5000) (Riemer et al., 1995); and mouse anti- $\alpha$-tubulin (1:25,000; Sigma-Aldrich). HRP-conjugated secondary antibodies (Jackson ImmunoResearch) were used at 1:10,000 (anti-mouse) or 1:20,000 (anti-rabbit). Blots were developed using the SuperSignal West Dura Extended Duration Substrate Kit (Thermo Scientific).

Fluorescence intensity measurement. Fluorescence from the neuronal marker anti-HRP was used to generate a region of interest (ROI) around the synaptic terminal using the thresholding function. Integrated fluorescent intensity was then measured within the ROI in arbitrary units and divided by the area of the ROI. MetaMorph software (Molecular Devices) was used for all measurements and analyzed using ANOVA followed by a Bonferroni post-comparison test or Student's $t$ test analysis (GraphPad Prism). All histograms and measurements are shown as mean \pm SEM.

\section{Results}

Photoreceptor neurotransmission defects in imp $\beta 11$ mutants A lethal complementation group consisting of five imp $\beta 11 \mathrm{mu}-$ tant alleles $(70,9,24,20$, and 202) was isolated in a forward genetic screen that has previously yielded a collection of mutants with defective synaptic development and transmission (Stowers et al., 2002; Dickman et al., 2005, 2008; Pack-Chung et al., 2007). This screen examined flies whose eyes were completely homozygous for a mutated chromosome arm $2 \mathrm{R}$ while the rest of the fly was heterozygous for that chromosome (Stowers and Schwarz, 1999). A primary screen selected flies that failed to perform phototaxis, and was followed by a secondary screen for abnormalities in the ERG. Each allele of the complementation group described here failed to complement the x-ray-induced deficiency $D f(2 R) \Delta M 22-103$ (hereafter referred to as $D f$; a generous gift from W. Boll and M. Noll). With partially overlapping deficiencies (Fig. 1A), the location of the alleles was restricted to an area bordered by the genes vha36 and poxn in the cytological region 52C. Sequencing of candidate genes revealed nonsense mutations in all five alleles in imp $\beta 11$, whose closest homologs include human importin- $\beta 11$ and yeast Kap120p/Lph2p sharing $34 \%$ and $23 \%$ amino acid identity, respectively (Plafker and Macara, 2000; Caesar et al., 2006). The sequence of imp $\beta 11$ predicts a protein of 1075 aa. At the $\mathrm{N}$ terminus, it possesses a conserved Ran-binding domain characteristic of all importin- $\beta$ s (Fig. $1 B, \mathrm{RBD}$ ). This region is followed by a long C-terminal region that shares homology only with the $\beta 11$ class of importins. Three alleles introduced a stop codon before or just at the beginning of the Ran-binding domain (Fig. $1 B$ ). Given that the Ranbinding domain is essential for nuclear import and most of the protein is absent, these are likely null alleles. The remaining two alleles, although independently derived, introduced stop codons at the same position, amino acid 853 (Fig. $1 B$ ). All alleles, when placed over the deficiency $(D f)$, failed to produce a full-length protein (Fig. $1 E$ ).

When these alleles were made homozygous selectively in the eye (Stowers and Schwarz, 1999), their ERGs lacked "on" and "off" transients in response to brief exposures to light (Fig. 1C, arrows). These transients are reported to arise from the synchronous activation of second-order cells in the visual system (Coombe, 1986); their absence indicates a failure of normal transmission by the photoreceptors. In contrast, the persistence of the sustained negative phase of the response indicates that the phototransduction cascade remained intact (Heisenberg, 1971). The four additional alleles displayed a similar ERG profile (data not shown; $n \geq 4$ ). Thus, the imp $\beta 11$ mutants were defective at a step downstream of the depolarization of the photoreceptor but 
upstream of activating the target neurons. We were able to fully restore the on and off transients by specifically expressing a $U A S$-imp $\beta 11-e G F P$ transgene in the photoreceptors by means of ey-GAL4 (Fig. 1C) (Brand and Perrimon, 1993; Hazelett et al., 1998; Stowers and Schwarz, 1999). The successful rescue indicates that the ERG defect resulted from loss of importin- $\beta 11$ and not from potential second-site mutations.

The ERG defect could have arisen from a functional defect in transmission or a developmental defect in the connections made by the photoreceptors. To analyze the photoreceptor axon projections, adult heads with homozygous mutant eyes were sectioned at $10 \mu \mathrm{m}$ with a cryostat, and photoreceptors were immunolabeled with a photoreceptor-specific antibody against Chaoptin. No gross abnormalities were detected in these projections: R1-R6 endings appeared to terminate appropriately in the lamina, and normal terminals appeared in the layers of the medulla that receive the endings of photoreceptors R7 and R8 (Fig. $1 D)$. In electron micrographs of the lamina from animals with homozygous mutant photoreceptors $\left(y, w ; F R T 42 D\right.$, imp $\beta 11^{70} /$ FRT42D, GMR-hid, cl;EGUF/+), the ordered structure of the cartridges containing endings of the R1-R6 photoreceptors appeared normal, and synapses were identified with appropriate T-bar ribbons, presynaptic densities, and clusters of vesicles. Small clear vesicles were normal in size but more numerous at mutant terminals (supplemental Fig. S1 $A, B$, available at www.jneurosci.org as supplemental material), a phenotype consistent with a decrease in vesicle release (Hiesinger et al., 2006). The cross-sectional area of the terminal profile was also significantly increased resulting in normal vesicle density (supplemental Fig. $\mathrm{S} 1 C, D$, available at www.jneurosci.org as supplemental material). In addition, capitate projections were almost absent from mutant terminals, a phenotype that has been interpreted previously to result from decreased endocytosis, a likely consequence of decreased vesicle release (supplemental Fig. S1E, available at www.jneurosci.org as supplemental material) (Fabian-Fine et al., 2003).

\section{Importin- $\beta 11$ expression in the larval nervous system}

Each imp $\beta 11$ allele, when placed over the $D f$, was lethal at the pharate adult stage, indicating that its function was not restricted to photoreceptors. To determine the developmental profile of importin- $\beta 11$, we generated an antiserum (5157) to amino acids 923 to 1075 (Fig. 1 B). On immunoblots of homogenates of thirdinstar larvae, this antiserum detected a band of the predicted molecular weight, $110 \mathrm{kDa}$, that was absent from imp $\beta 11^{70} / \mathrm{Df}$ (Fig. $1 E$ ). Nonspecific bands of 70 and $60 \mathrm{kDa}$ were also observed that could not be removed by affinity purification.
B

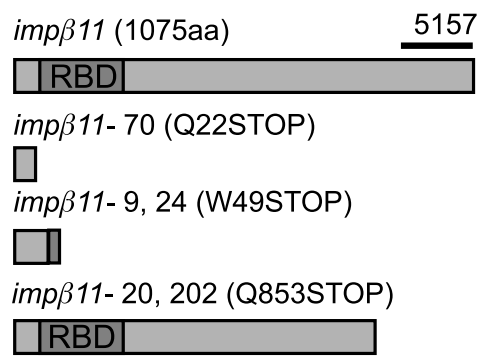

D
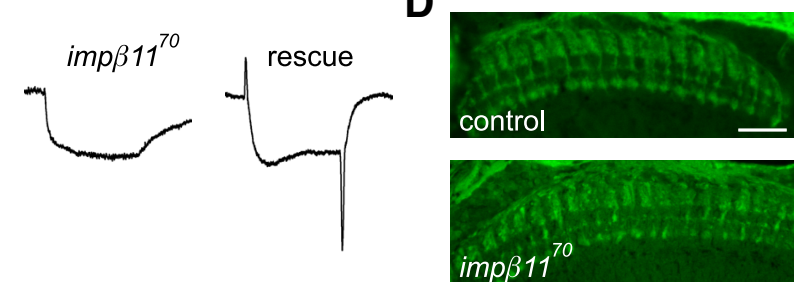

$\operatorname{imp} \beta 11$

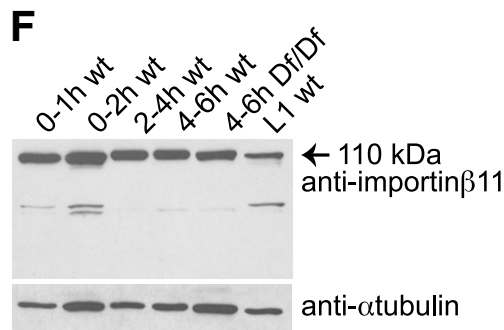

$-\infty-$ anti-atubulin

Figure 1. imp $\beta 11$ mutants have defective photoreceptor neurotransmission but normal projections. $\boldsymbol{A}$, The genomic region of complementation group isolated in the EGUF-hid screen was narrowed using two overlapping deficiencies, Df(2R) $\Delta M 22-103$ $d D f(2 R) J P 5$, which contain the indicated predicted open reading frames, including the gene ran-binding protein 11 or importin(gray). $\boldsymbol{B}$, Schematic of wild-type importin- $\beta 11$ with the Ran-binding domain (RBD) marked, and the predicted products of antibody 5157 was raised against the C-terminal region (bar). C Representative responses to light (between arrows) from control ( $y$,w; FRT42D, GMR-hid 2R, CL/Cy0; EGUF), imp $\beta 11^{70}$ mutant eyes ( $y, w ; F R T 42 D$, GMR-hid 2R, CL/FRT42D, imp $\beta 11^{70}$; EGUF), and mutant eyes expressing a UAS-imp $\beta 11$-eGFP transgene $\left(y, w ; F R T 42 D, G M R\right.$-hid 2R, CL/FRT42D, imp $\beta 11^{70}$; EGUF/UAS$D f(2 R) \Delta M 22-103$ homozygous nulls (Df/Df).

Importin- $\beta 11$ protein was detected throughout the life cycle in wild-type animals, including lysates from embryos aged 0 to $2 \mathrm{~h}$ after egg deposition, a stage before initiation of zygotic transcription (Fig. 1F) (Anderson and Lengyel, 1979). In addition, lysates of homozygous $D f$ embryos had a detectable band at 110 $\mathrm{kDa}$ (Fig. $1 F$ ). Because this deficiency removes the entire imp $\beta 11$ gene, this early expression must result from maternally contributed mRNA or protein. We reasoned that maternally derived importin- $\beta 11$ might mask the requirements for this protein before the pupal stage. Therefore, we used the ovo ${ }^{\mathrm{D}}$ method (Chou and Perrimon, 1992, 1996) to generate maternal germline clones homozygous for the $i m p \beta 11^{70}$ and $i m p \beta 11^{20}$ alleles. Mutant females did not lay eggs, and on examination of mutant ovarioles, the ovo ${ }^{\mathrm{D}}$ phenotype was evident, consistent with the hypothesis that importin- $\beta 11$ plays an essential role in the female germline (Chan et al., 2008). Hereafter, unless otherwise notated, late third-instar larvae of genotype $y, w ; F R T 42 D$, imp $\beta 11^{70} / D f$ were used for experiments and will be referred to as imp $\beta 11$ null lar- 
vae. At this stage, maternally supplied importin- $\beta 11$ was no longer detectable by Western blot in this genotype (Fig. $1 E$ ).

Third-instar larvae offered us the advantage of using homozygous mutant animals to characterize the phenotype at the NMJ, a synapse amenable to both anatomical and electrophysiological analysis (Schwarz, 2006). Identifiable motor neurons exit the larval ventral nerve cord (VNC) and innervate specific muscles in each hemisegment of the body wall, where they form synaptic varicosities or boutons (Keshishian et al., 1996).

To examine importin- $\beta 11$ immunoreactivity at these NMJs, body wall preparations were labeled with antiserum 5157 (Fig. $2 A-F)$. In control body walls, muscle nuclei were immunoreactive, and the protein was enriched in puncta on the nuclear envelope, presumably corresponding to nuclear pores (Fig. $2 B$ ). This immunoreactivity was absent in imp $\beta 11$ mutants (Fig. $2 C, D$ ). The antibody also labeled the NMJ (Fig. $2 A, E$ ), but this signal was not caused exclusively by importin- $\beta 11$ because it was also present at mutant NMJs (Fig. 2C,F). To circumvent this problem, we created a transgenic line (UAS-imp $\beta 11-e G F P)$ expressing importin- $\beta 11$ fused to eGFP and placed under the control of a UAS promoter (Brand and Perrimon, 1993). When expressed either ubiquitously (with a $d a-G A L 4$ driver) (Wodarz et al., 1995) or exclusively in the nervous system (with an elavGAL4 driver) (Luo et al., 1994), but not when expressed in muscle (with a G14-GAL4 driver) (Aberle et al., 2002), this construct rescued the lethality of $i m p \beta 11$ null larvae, indicating both that the transgene was functional and that lethality at the pupal stage was attributable to an essential neuronal function of importin- $\beta 11$.

In the nervous system, importin- $\beta 11-e G F P$ protein localized to puncta in axons, to the cytoplasm and nuclei of many cells in the VNC, including motor neurons, and in the neuropil, the synaptic region of the VNC (Fig. $2 G-I$ ). As expected for a nuclear import factor, at high magnification, importin- $\beta 11$-eGFP was observed at the nuclear envelope of these neurons where it concentrated at nuclear pores (Fig. $2 J$ ). Consistent with the importin-eGFP signal in nerves and the synaptic neuropil, neuronally expressed importin- $\beta 11-e G F P$ was also present at presynaptic neuromuscular terminals (Fig. $2 K, L$ ). The fluorescent importin- $\beta 11$, however, was only prominent in $\sim 30 \%$ of body wall NMJs in any given larva, and filled some but not all boutons within a given synapse (Fig. $2 \mathrm{~L}$ ). This irregular distribution likely reflects the pattern of expression of the GAL4 line elav-GAL4, the insertion site of the UAS-imp $\beta 11-e G F P$ transgene, or a combination of both. A stronger, ubiquitous driver (tubulin-GAL4) (Lee and Luo, 1999) caused importin-eGFP to be visible in all boutons within a given NMJ (Fig. $2 M$ ). Furthermore, because the $\mathrm{NMJ}$ at muscles $6 / 7$ contains the terminals of two axons, we also examined the simpler, singly innervated muscle 4 (Fig. $2 \mathrm{~N}$ ) (Lnenicka and Keshishian, 2000; Hoang and Chiba, 2001). Here too, all boutons were positive for eGFP. These data suggest that importin- $\beta 11$ can localize to synaptic terminals in addition to the synapse-rich region of the VNC, nuclear pores, cell bodies, and muscle nuclei. We also observed importin- $\beta 11$-eGFP localization to muscle nuclei and trachea when expressed ubiquitously (Fig. $2 M, N$ ). To address whether endogenous importin- $\beta 11$ could also localize to synapses, we performed a subcellular fractionation of adult head lysates (Kelly, 1983). As expected, importin- $\beta 11$ immunoreactivity was detected in the nuclear fraction, on long exposure, but it also appeared in the synaptosome fraction and in the cytosol (supplemental Fig. S2, available at www.jneurosci.org as supplemental material). Thus, importin$\beta 11$ is likely to mediate import through nuclear pores in both neurons and muscle cells, but may also have a function at synaptic terminals.

\section{Structural and functional defects at the imp $\beta 11$ mutant NMJ}

Because we could detect a synaptic pool of importin- $\beta 11$, we next examined the NMJ for potential structural and developmental phenotypes by counting the number of boutons present at the $\mathrm{NMJ}$ in wild-type and imp $\beta 11$ mutant larvae. Although the size of their muscle fibers was normal, imp $\beta 11$ mutant larvae had a $30 \%$ reduction in bouton number when compared to either control larvae or heterozygote mutant larvae (Fig. $3 A, B$ ). Bouton counts are frequently expressed normalized to muscle surface because bouton number is known to scale with muscle size during development (Lnenicka and Keshishian, 2000; Marqués et al., 2002). Thus, normalized to account for variations in larval size, imp $\beta 11$ null mutants showed a similar 30\% decrease in bouton density (Fig. 3B). Synaptic footprints, a sign that boutons have retracted (Eaton et al., 2002), were scant in both mutant and control (control, zero footprints in $\sim 300$ boutons; imp $\beta 11$, two footprints in $\sim 500$ boutons). Therefore, the altered bouton number likely reflects a reduction in bouton addition as the terminal developed.

Inputs from both type Ib (large) and type Is (small) boutons contribute to the synaptic arbor and electrophysiological profile (Johansen et al., 1989; Lnenicka and Keshishian, 2000). There was no preferential loss of Ib or Is boutons in imp $\beta 11$ NMJs, but arbor length and bouton diameter were both slightly decreased when compared to control (data not shown). In addition, the geometry of the synapse was altered in imp $\beta 11$ mutants with axonal branches remaining close to the intermuscular cleft rather than spreading across the width of the muscle (Fig. $3 A$ ).

To determine whether the reduction in bouton number was primarily the result of presynaptic or postsynaptic defects, we expressed UAS-imp $\beta 11-e G F P$ in all tissues (with a da-GAL4 driver), in neurons (with an elav-GAL4 driver), or in muscle (with a G14-GAL4 driver). The reduction in bouton number/ muscle surface area was statistically rescued to control values when imp $\beta 11-e G F P$ was expressed in all cells and in neurons alone, but not by expression in muscle alone (Fig. $3 B$ ) (control, $0.88 \pm 0.048 / 1000 \mu \mathrm{m}^{2} ; i m p \beta 11^{70} / D f, 0.62 \pm 0.044 / 1000 \mu \mathrm{m}^{2}$; all cells rescue, $0.93 \pm 0.036 / 1000 \mu \mathrm{m}^{2}$; neuron rescue, $0.92 \pm$ $0.034 / 1000 \mu \mathrm{m}^{2}$; muscle rescue, $0.61 \pm 0.029 / 1000 \mu \mathrm{m}^{2} ; n \geq 17$ for each genotype). Thus, importin- $\beta 11$ is primarily required presynaptically for normal bouton formation and arborization.

To determine whether these structural defects were accompanied by electrophysiological defects, we stimulated the motor nerve that innervates muscle 6 to evoke excitatory junctional potentials (EJPs). Consistent with the morphological defects, the amplitude of EJPs was reduced by $40 \%$ in the mutant larvae as compared with control (Fig. 3C,E) (control, $35.6 \pm 1.08 \mathrm{mV}$; imp $\beta 11,21.1 \pm 1.81 \mathrm{mV} ; n \geq 12$ for both genotypes). Vesicles also fuse spontaneously at the NMJ, giving rise to quantal events or miniature EJPs (mEJPs). mEJPs were also altered (Fig. $3 D, F, G)$; imp $\beta 11$ released vesicles at a lower frequency, about $65 \%$ less than control (Fig. $3 F$ ) (control, $3.34 \pm 0.367 \mathrm{~Hz}$; $\operatorname{imp} \beta 11,1.19 \pm 0.171 \mathrm{~Hz} ; n \geq 11$ for both genotypes). This decrease could, at least in part, reflect fewer release sites in the smaller synaptic arbor. There was also a small, but statistically significant, decrease in the amplitude of the mEJPs (Fig. $3 G$ ) (control, $0.95 \pm 0.052 \mathrm{mV}$; $i m p \beta 11,0.77 \pm 0.035 \mathrm{mV} ; n \geq 11$ for both genotypes), suggesting that imp $\beta 11$ mutants may have a mild reduction in postsynaptic receptor density or sensitivity (Petersen et al., 1997; DiAntonio et al., 1999). As calculated from these data, quantal content, or the number of vesicles released per 

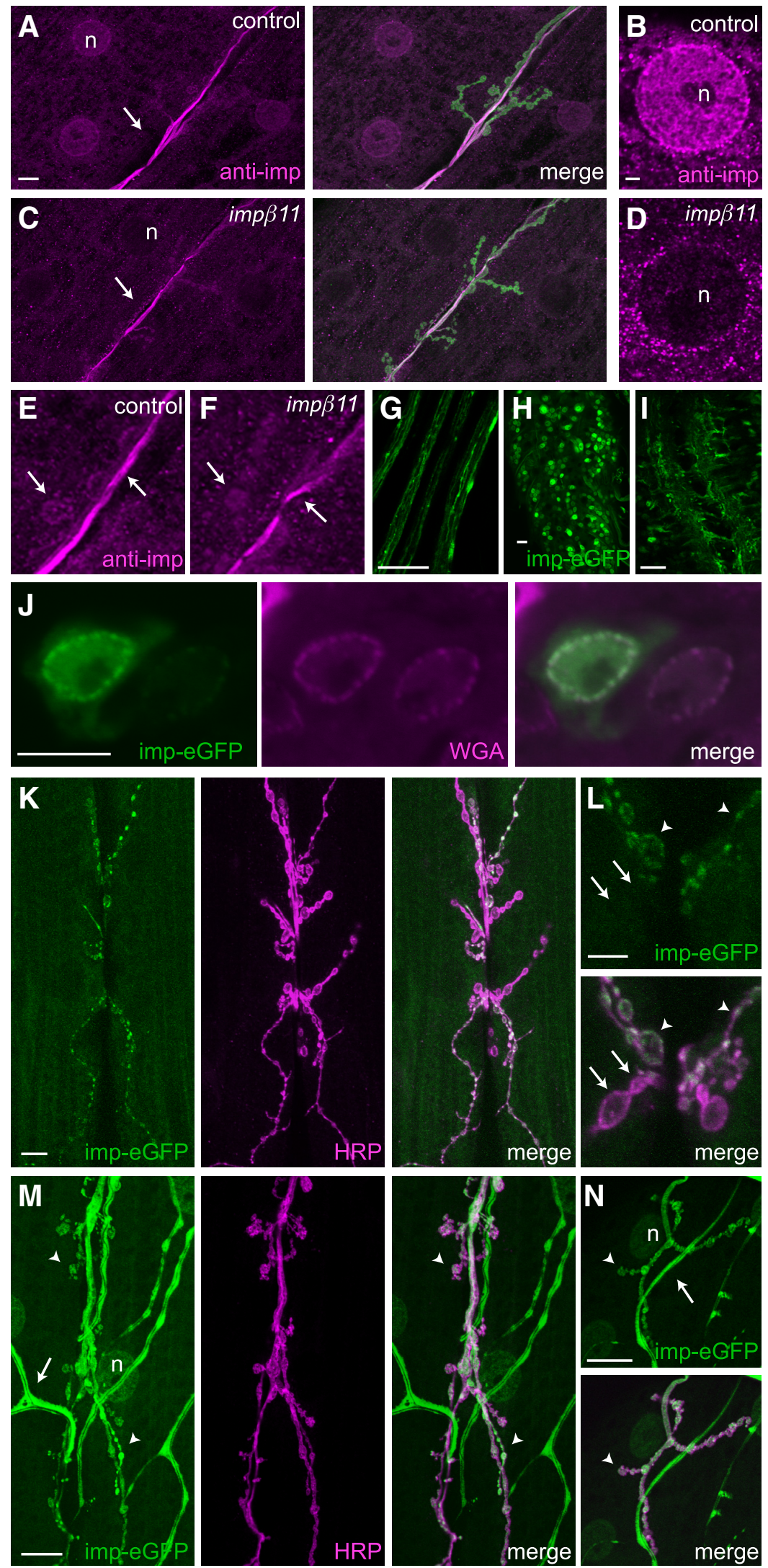

Figure 2. Importin- $\beta 11$ localization in third-instar larvae. $\boldsymbol{A}-\boldsymbol{F}$, Representative confocal images of NMJs and muscle fields immunostained with the rabbit antiserum 5157 against importin- $\beta 11$ (magenta) and the neuronal membrane marker anti-HRP (green). $\boldsymbol{A}, \boldsymbol{B}$, Immunoreactivity was present in control muscle nuclei (n) and enriched around the nuclear envelope, but was not in imp $\beta 11$ muscles $(\boldsymbol{C}, \boldsymbol{D}) . \boldsymbol{A}, \boldsymbol{C}, \boldsymbol{E}, \boldsymbol{F}$, At the NMJ, immunoreactivity unrelated to importin- $\beta 11$ was detected both in control and imp $\beta 11$ NMJs (arrows). G-J, UAS-imp $\beta 11$-eGFP expressed in the nervous system with elav-GAL4, localized to puncta in the action potential, was reduced by $30 \%$ in imp $\beta 11$ (Fig. $3 H$ ) (control, $43.3 \pm 2.40$; $\operatorname{imp} \beta 11,30.7 \pm 2.82 ; n \geq 10$ for both genotypes) when corrected for nonlinear summation (Martin, 1955).

Defects in synaptic transmission can result from either presynaptic or postsynaptic mechanisms. We therefore selectively restored function of importin- $\beta 11$ using UAS-imp $\beta 11-e G F P$ in either neurons or muscle. The observed defects in quantal content and EJP amplitude in imp $\beta 11$ were fully rescued with expression of the UAS-imp $\beta 11-e G F P$ transgene in the nervous system with elav-GAL4 [EJP amplitude: neuron rescue, $32.3 \pm$ $1.71 \mathrm{mV}$; muscle rescue, $27.1 \pm 1.11 \mathrm{mV}$ (Fig. $3 E$ ); quantal content: neuron rescue, $50.1 \pm 2.71 ;$ muscle rescue, $39.7 \pm 1.98$ (Fig. $3 H) ; n \geq 10$ for each genotype]. Although quantal content was somewhat increased by transgene expression in muscle, it did not correspond to a statistically significant restoration of EJP amplitude. In contrast, neuronal rescue was significant and complete, indicating that neuronal expression of importin- $\beta 11$ was both sufficient and necessary to rescue the electrophysiological defect ( $p$ values compared to control: neuron rescue, $p \geq 0.05$; muscle rescue, $p<0.0001$; compared to mutant: neuron rescue, $p<0.0001$; muscle rescue, $p \geq 0.05$ ). Separately, neither neuronal nor muscle expression of importin- $\beta 11-e G F P$ was adequate to restore mEJP frequency or amplitude to control levels; however, concurrent expression in both neurons and muscle significantly increased mini frequency (Fig. $3 F$ ) (neuron rescue, $1.64 \pm 0.250 \mathrm{~Hz}, 0.73 \pm 0.033 \mathrm{mV}$; muscle rescue, $1.55 \pm 0.314 \mathrm{~Hz}, 0.76 \pm$ $0.035 \mathrm{mV}$; all cells rescue, $3.02 \pm 0.546$ $\mathrm{Hz}, 0.64 \pm 0.025 \mathrm{mV} ; p \geq 0.05$ when $d a-$ GAL4 frequency was compared to control; $n \geq 11$ for each genotype). mEJP amplitude was not rescued, and may be partic-

$\leftarrow$

segmental nerves $(\boldsymbol{G})$, and in the VNC to neuronal cell bodies and nuclei $(\boldsymbol{H})$, neuropil $(\boldsymbol{I})$, and nuclear pores $(\boldsymbol{J})$. Nuclear pores were identified with fluorescently conjugated wheat germ agglutinin (magenta) (Finlay et al., 1987). $K$, $\boldsymbol{L}$, When driven with elav-GAL4, imp $\beta 11$-eGFP was present in most type $\mathrm{lb}$ and Is boutons (arrowheads) of the anti-HRP-stained NMJ, but was not detected in some (arrows). $\boldsymbol{M}, \boldsymbol{N}$, When importin-eGFP was driven with a stronger driver, tubulinGAL4, eGFP signal was visible in all boutons at muscles 6/7 ( $\boldsymbol{M}$, arrowheads) and 4 ( $\boldsymbol{N}$, arrowheads). eGFP signal was also detected in muscle nuclei (n) and trachea (arrows). Scale bars: $\boldsymbol{A}$ (for $\boldsymbol{A}, \boldsymbol{C}$ ), G-I, $\boldsymbol{K}, \boldsymbol{M}, \boldsymbol{N}, 20 \mu \mathrm{m} ; \boldsymbol{B}$ (for $\boldsymbol{B}, \boldsymbol{D}$ ), J, $\boldsymbol{L}, 5 \mu \mathrm{m}$. Genotypes are as follows: $\boldsymbol{A}-\boldsymbol{F}, y, w ; F R T 42 D ; \boldsymbol{G}-\boldsymbol{L}$, FRT42D, imp $\beta 11^{70} /+$; elav-GAL4/UAS-imp $\beta 11$-eGFP; $\boldsymbol{M}, \mathbf{N}$, FRT42D, imp $\beta 11^{70} / D f$; tubulin-GAL4/UAS-imp $\beta 11$-eGFP. 


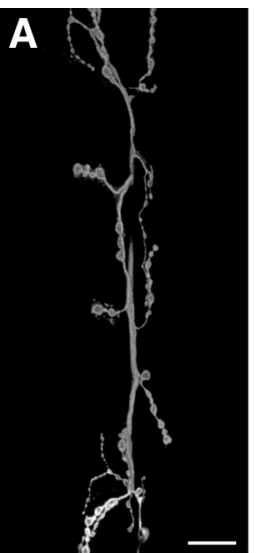

control

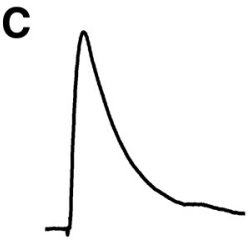
control

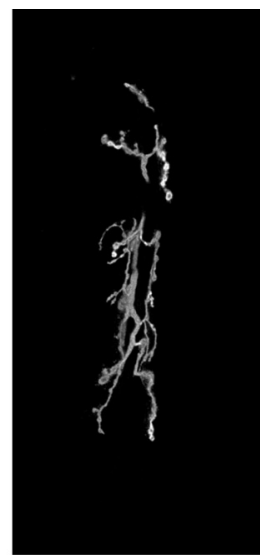

$\operatorname{imp} \beta 11$
B

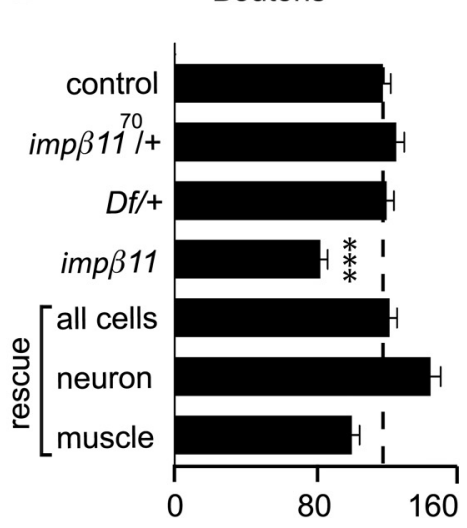

Muscle SA $\left(\times 10^{5} \mu \mathrm{m}^{2}\right)$

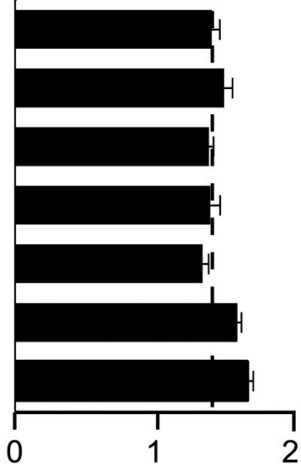

Boutons / $1000 \mu \mathrm{m}^{2} \mathrm{SA}$

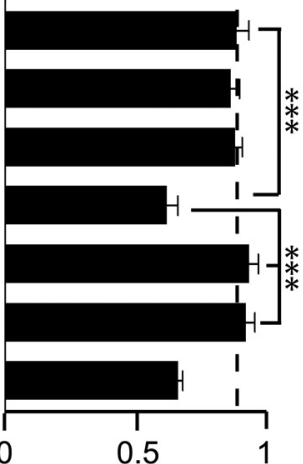

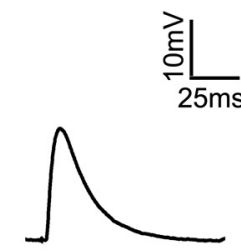

imp $\beta 11$

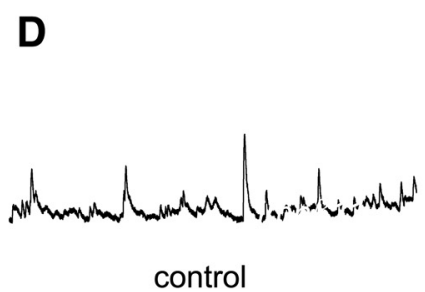

G

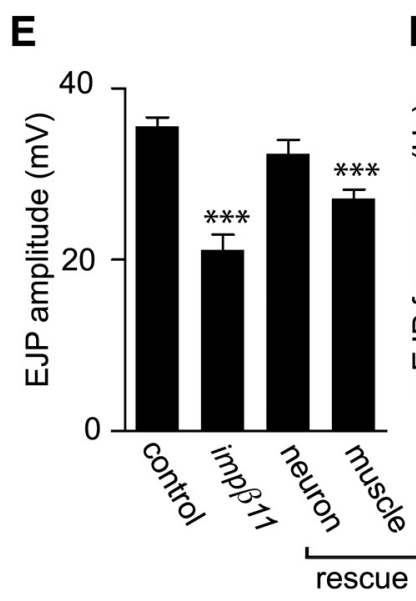

$\mathbf{F}$
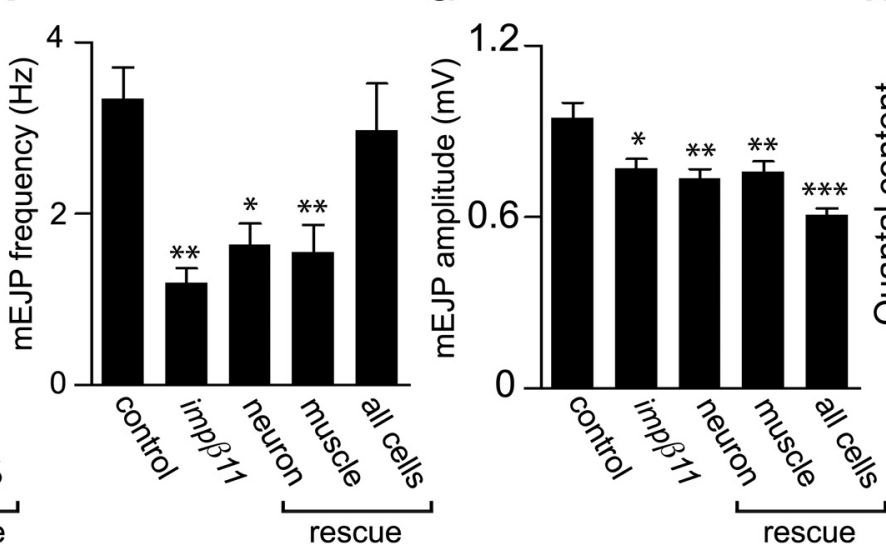

H

Figure 3. Reduced bouton number and quantal content in imp $\beta 11$ mutants. $\boldsymbol{A}$, Representative confocal images of third-instar larval NMJs stained with anti-HRP. The synaptic arbor in imp $\beta 11$ mutant larvae was shorter and narrower. Scale bar, $20 \mu \mathrm{m}$. $\boldsymbol{B}$, Bouton number, muscle surface area, and bouton number normalized to muscle area were calculated for all genotypes. Although muscle size was normal, imp $\beta 11$ muscles had fewer boutons than control or heterozygous animals. Bouton number was rescued with UAS-imp $\beta 11$-eGFP transgene expression in all cells (da-GAL4) or in neurons (elav-GAL4), but not by expression in muscle (G14-GAL4). The slight increases in bouton number and muscle size observed with the G14-GAL4 driver were not seen with 24B-GAL4, another muscle driver. $\boldsymbol{C}, \boldsymbol{D}$, Representative EJPs and mEJPs from control and imp $\beta 11$ mutant larvae. $\boldsymbol{E}$, EJP amplitude was reduced in imp $\beta 11$ and fully rescued by transgene expression in neurons but not by expression in muscle. $\boldsymbol{F}$, mEJP frequency was decreased in imp $\beta 11$ and was only fully rescued with imp $\beta 11$ transgene expression in both presynaptic and postsynaptic compartments with da-GAL4. G, mEJP amplitude was slightly reduced in imp $\beta 11$, but was not restored by transgene expression. $\boldsymbol{H}$, Quantal content, corrected for nonlinear summation, was reduced in imp $\beta 11$ and rescued by transgene expression in neurons. Recordings were conducted in $1 \mathrm{mM} \mathrm{Ca}{ }^{2+} \mathrm{HL}^{*}{ }^{*} p<0.05$; ${ }^{* *} p<0.001$; ${ }^{* * *} p<0.0001$ compared to control. Genotypes are as follows: control ( $y, w_{\text {; }}$ FRT42D), imp $\beta 11$ (FRT42D, imp $\beta 11^{70} / D f$ ), all cells (FRT42D, imp $\beta 11^{70} / D f ;$ da-GAL4/UAS-imp $\beta 11$-eGFP), neuron rescue (FRT42D, imp $\beta 11^{70} / D f ;$ elav-GAL4/UAS-imp $\beta 11$-eGFP), muscle rescue (FRT42D imp $\beta 11^{70}$, G14-GAL4/Df; UAS-imp $\beta 11$-eGFP/+).

ularly sensitive to appropriate expression levels of the importin. Thus, presynaptic importin- $\beta 11$ was sufficient to restore bouton number and EJP amplitude, suggesting that importin- $\beta 11$ at the presynapse was required for normal NMJ arborization and function.

To further characterize imp $\beta 11$ abnormalities at the NMJ, we examined proteins of known importance to the development and function of this synapse. Several structural components were investigated that could potentially contribute to the phenotype. However, both the cell-adhesion molecule FasII and the microtubuleassociated protein Futsch (Schuster et al., 1996; Hummel et al., 2000; Roos et al., 2000) localized normally in imp $\beta 11$ mutant
NMJs (supplemental Fig. S3, available at www.jneurosci.org as supplemental material). The reduction in bouton number was therefore not likely to arise from gross abnormalities in these structural components. Similarly, Syt I immunolabeling indicated that synaptic vesicles were concentrated within boutons (Fig. 4A) (DiAntonio et al., 1993) and contained slightly more Syt I reactivity as measured by fluorescence intensity (supplemental Fig. S4, available at www.jneurosci.org as supplemental material). NMJs were also labeled with the monoclonal antibody nc82, which recognizes the active zone component BRP (Kittel et al., 2006; Wagh et al., 2006; Hofbauer et al., 2009). The density and number of BRP puncta per bouton in imp $\beta 11$ mutant NMJs were 
Table 1. Quantification of glutamate receptors in third instar control and imp- $\beta 11$ larvae

\begin{tabular}{llll}
\hline Genotype & GluRIIA & GluRIIB & GluRIIC \\
\hline Control & $0.63 \pm 0.030$ & $0.54 \pm 0.018$ & $0.52 \pm 0.025$ \\
imp- $\beta 11^{70} / D f$ & $0.65 \pm 0.036$ & $0.54 \pm 0.039$ & $0.58 \pm 0.030$
\end{tabular}

Quantification of the number of glutamate receptor puncta at synaptic boutons on muscle 4 divided by the synaptic surface area as determined by HRP staining. $n>12$ NMJs for both genotypes.

unchanged (Fig. $4 B$ ) (control, $0.70 \pm 0.029$ puncta/ $\mu \mathrm{m}^{2} ;$ imp $\beta 11^{70} /$ $D f, 0.79 \pm 0.035$ puncta $/ \mu \mathrm{m}^{2} ; n \geq 13$ for both genotypes). However, because there were fewer boutons per NMJ, there was a net decrease in total active zone number, and this loss of release sites was likely an important factor in the reduction in synaptic strength.

The impairment in mEJP amplitude suggested that there might have been an alteration in postsynaptic glutamate receptors (Petersen et al., 1997; DiAntonio et al., 1999; Marrus et al., 2004). To examine receptor density and localization, we therefore undertook immunolabeling for GluRIIA, GluRIIB, and GluRIIC, but all appeared normal in the imp $\beta 11$ mutant NMJs. The intensity of the labeling was unchanged and clusters of receptors properly localized beneath active zones (Fig. 4C, Table 1). Thus, within the reduced synaptic arbor of imp $\beta 11$ mutants, each bouton retained active zones with synaptic vesicles and faced a postsynaptic membrane with properly clustered glutamate receptors.

\section{Reduced pMAD at imp $\beta 11$ NMJs}

The quantitative reduction in bouton number suggested that imp $\beta 11$ mutants may be defective in one of several signaling pathways known to modulate the development of this synapse (Collins and DiAntonio, 2007). These include the BMP pathway, the Wnt pathway, and the mitogen-activated protein kinase pathways (Marqués, 2005; Collins et al., 2006; Speese and Budnik, 2007). In particular, disruption of the BMP pathway reduces bouton number and EJP amplitude, and thus resembles the imp $\beta 11$ phenotype. At the NMJ, secretion of the ligand glass bottom boat (Gbb) from the muscle activates the BMP pathway by causing the receptor complex composed of Wit, Tkv, and Saxophone (Sax) to phosphorylate the signaling molecule MAD (Keshishian and Kim, 2004). The C-terminally phosphorylated form, pMAD, is abundant both at the NMJ and in motor neuron nuclei, and therefore may act locally or by retrogradely translocating to the nucleus (Allan et al., 2003; McCabe et al., 2003; Marqués, 2005). The pathway is likely to function bidirectionally, with some pMAD generated in the muscle as well as in the nerve terminals (Dudu et al., 2006; Collins and DiAntonio, 2007). Recently, several studies have identified proteins that interact with and modulate the BMP pathway at the Drosophila NMJ (Wang et al., 2007; O'Connor-Giles et al., 2008; Merino et al., 2009). We therefore examined the distribution of $\mathrm{pMAD}$ as a readout of the activity of this pathway and its potential interaction with importin- $\beta 11$.

In control animals, pMAD immunoreactivity (Persson et al., 1998) was present both at the NMJ, overlapping with the neuronal marker HRP, and in motoneuron nuclei (Fig. $4 D, F$ ). In imp $\beta 11$ mutants, pMAD still accumulated in motor neuron nuclei (Fig. $4 G$ ), but synaptic pMAD was markedly reduced at the NMJs of all muscles (Fig. $4 E$; quantified in supplemental Fig. S5, available at www.jneurosci.org as supplemental material). This phenotype is distinct from that of wit mutants, which lose both nuclear (Marqués et al., 2002) and synaptic pMAD (sup- plemental Fig. S6, available at www.jneurosci.org as supplemental material).

Like the localization of pMAD itself (Dudu et al., 2006; Collins and DiAntonio, 2007), the reduction in synaptic pMAD in imp $\beta 11$ appeared to have both presynaptic and postsynaptic components. Restoration of importin- $\beta 11$ expression to the nervous system, by means of elav-GAL4 or ubiquitous expression with $d a-G A L 4$, was sufficient to restore pMAD immunolabeling to the nerve terminals to control levels (Fig. $4 H$, I; quantified in supplemental Fig. S5, available at www.jneurosci.org as supplemental material). Expression of the transgene in muscle with G14-GAL4, however, was also capable of increasing pMAD immunoreactivity to the mutant NMJ, but to a lesser extent than neuronal or ubiquitous rescue (Fig. 4J; quantified in supplemental Fig. S5, available at www.jneurosci.org as supplemental material). The NMJ phenotypes of MAD mutants and other genes in the BMP signaling pathway are known to arise from presynaptic requirements for this pathway (Aberle et al., 2002; McCabe et al., 2004). Because bouton number, EJP amplitude, and pMAD phenotypes of $i m p \beta 11$ were rescued to control values with presynaptic restoration of importin- $\beta 11$, loss of specifically presynaptic pMAD is also the most likely explanation of the anatomical and electrophysiological defects in imp $\beta 11$.

\section{BMP pathway components are present at imp $\beta 11$ mutant NMJs}

Importins, by mediating the nuclear import of transcription factors, might be required for the expression of a component of the BMP pathway, and thus influence the production of pMAD. We therefore investigated whether the loss of synaptic pMAD resulted from the loss of a component of the pathway. To test whether low Gbb production caused the reduced pMAD, we expressed a $U A S$ - $g b b$ transgene in muscle and determined whether it would restore PMAD to the mutant NMJ. Although expression of the $g b b$ transgene increased nuclear pMAD in muscle, thereby confirming the efficacy of the transgene, pMAD was not restored to imp $\beta 11$ mutant NMJs (Fig. $5 A-E$ ). Thus, loss of Gbb is unlikely to explain the loss of pMAD in imp $\beta 11$ mutants. Reductions in synaptic pMAD might also have arisen because of the absence of the receptors or MAD itself. We addressed these possibilities by comparing the immunolocalization of MAD, Wit, and Tkv. MAD and Wit labeling in control NMJs were detected both within boutons and at their margins; the latter staining likely represented both presynaptic and postsynaptic pools. AntiTkv clearly labeled both the presynaptic and postsynaptic compartments. Similar patterns of immunolabeling for MAD, Wit, and Tkv were also observed in imp $\beta 11$ NMJs (Fig. $5 F-K$ ), indicating that the loss of pMAD from the NMJ was not caused by the absence of the substrate or receptors from the synapse. Because anti-MAD and anti-Wit only dimly labeled some boutons, we used additional methods to examine the presence of each protein in imp $\beta 11$. For Wit, we examined the VNC where the receptor had previously been localized (Aberle et al., 2002) and found that anti-Wit immunoreactivity was not markedly altered in imp $\beta 11$ (supplemental Fig. S7 $A, B$, available at www.jneurosci.org as supplemental material). As an additional method to test whether a lack of MAD could account for the loss of pMAD, we expressed a $U A S-G F P-M A D$ transgene in the nervous system of imp $\beta 11 \mathrm{mu}-$ tant larvae with elav-GAL4. This transgene can functionally replace the endogenous protein in MAD mutants (Dudu et al., 2006). Expression of UAS-GFP-MAD, however, did not rescue the pMAD defect in $i m p \beta 11$, further indicating that the mutant defect was not attributable to a reduction in the substrate MAD 

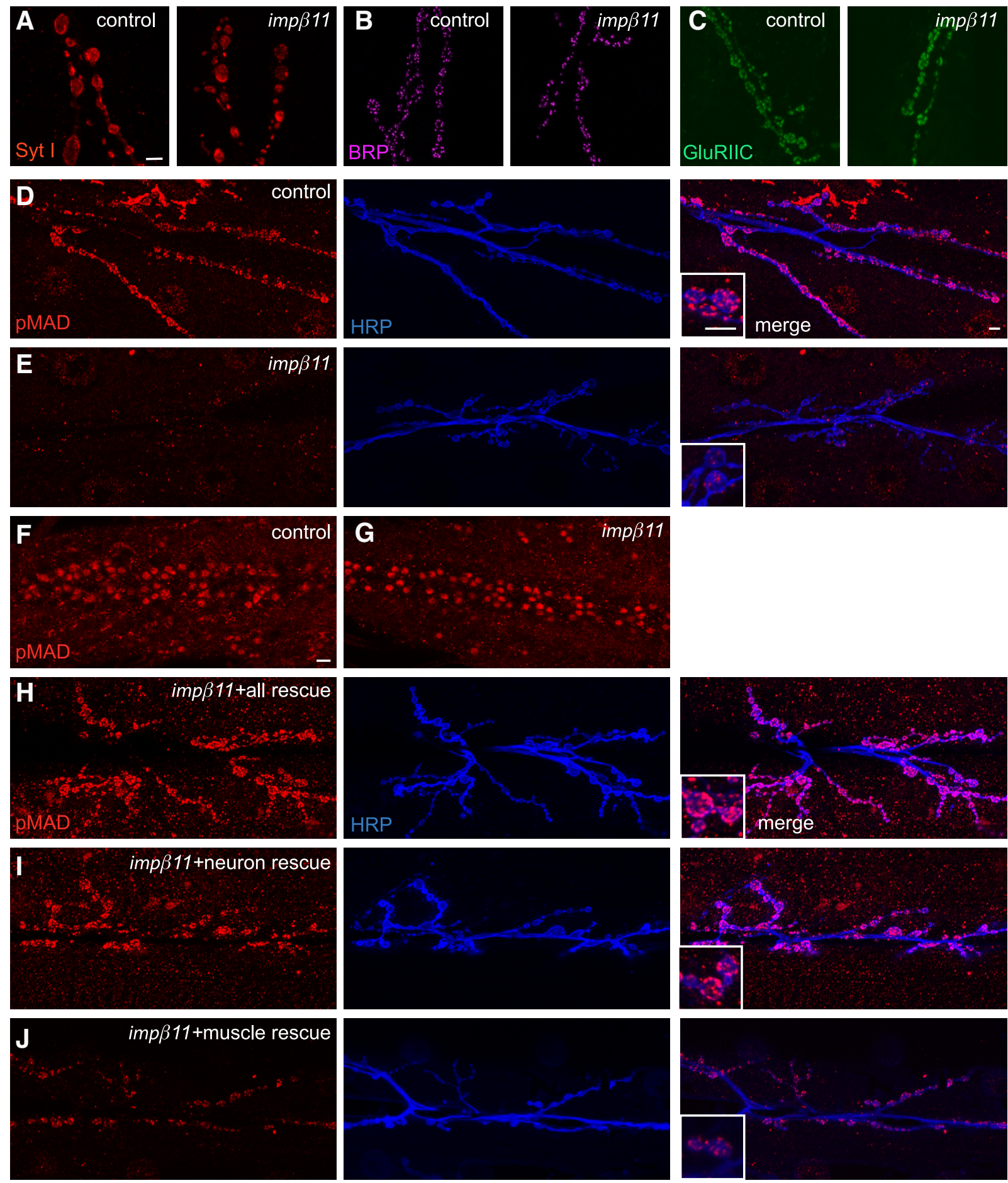

Figure 4. Synaptic pMAD is reduced in imp $\beta 11$ mutants but other synaptic proteins appear normal. $A-C$, Representative confocal images of third-instar NMJs stained with antibodies to synaptic proteins to localize synaptic vesicles (with anti-Sytl; $\boldsymbol{A}$, red), active zones (with anti-BRP; $\boldsymbol{B}$, magenta), and glutamate receptors (with anti-GluRIIC; $\boldsymbol{C}$, green) to control and imp $\beta 11$ mutant terminals. These proteins appeared unaltered at the imp $\beta 11$ mutant NMJs. $\boldsymbol{D}, \boldsymbol{E}$, Immunoreactivity for pMAD (red) at control (D) and mutant (E) NMJs whose neurons were marked with anti-HRP (blue). imp $\beta 11$ mutants consistently had low levels of pMAD at the NMJ. Insets show higher magnifications of the representative boutons. $F, G$, Nuclear pMAD was detected in both control and mutant VNCs including motor neurons. $\boldsymbol{H}-\boldsymbol{J}$, Expression of UAS-imp $\beta 11$-eGFP in the imp $\beta 11$ mutant background in all cells $(\boldsymbol{H})$ or in neurons $(\boldsymbol{I})$ rescued pMAD immunoreactivity (red) to control levels, but expression in muscle $(\boldsymbol{J})$ only slightly increased pMAD staining above the levels in the mutant. Scale bars, $10 \mu \mathrm{m}$. Genotypes are as follows: control $(y, w ; F R T 42 D), i m p \beta 11$ (FRT42D, imp $\left.\beta 11^{70} / D f\right)$, all rescue (FRT42D, imp $\beta 11^{70} / D f$; da-GAL4/UAS-imp $\left.\beta 11-e G F P\right)$, neuron rescue (FRT42D, imp $\beta 11^{70} / D f ;$ elav-GAL4/UAS-imp $\left.\beta 11-e G F P\right)$, muscle rescue (FRT42D, imp $\beta 17^{70}$, G14-GAL4/Df; UAS-imp $\beta 11-e G F P /+)$.

(supplemental Fig. S7C-F, available at www.jneurosci.org as supplemental material). Thus, in imp $\beta 11$ mutants, the reduction in synaptic pMAD could not be explained by a loss of the ligand, receptors, or MAD itself.
To determine whether other functions that rely on BMP signaling were intact in imp $\beta 11$ mutants, philanthotoxin-induced synaptic homeostasis was examined. This phenomenon entails a compensatory presynaptic increase in quantal content after the 
Table 2. Genetic interactions between imp $\beta 11$ and BMP components

\begin{tabular}{|c|c|c|}
\hline Genotype & Mean bouton no. & $\%$ of control \\
\hline Control & $0.88 \pm 0.048$ & 100 \\
\hline $\operatorname{imp} \beta 11^{70} /+$ & $0.86 \pm 0.033$ & 98 \\
\hline$D f /+$ & $0.88 \pm 0.028$ & 100 \\
\hline $\operatorname{imp} \beta 11^{70} / D f$ & $0.62 \pm 0.044$ & 70 \\
\hline $\operatorname{mad}^{10} /+$ & $0.92 \pm 0.046$ & 104 \\
\hline wit $^{A 12} /+$ & $0.87 \pm 0.057$ & 99 \\
\hline$w_{i t}^{H A 1} /+$ & $0.92 \pm 0.040$ & 104 \\
\hline$w_{i t}^{A 12} / w_{i t}^{B 11}$ & $0.42 \pm 0.047$ & 48 \\
\hline $\operatorname{imp} \beta 11^{70} / \mathrm{mad}^{10}$ & $0.75 \pm 0.020$ & 85 \\
\hline $\operatorname{imp} \beta 11^{70} /+;$ wit $^{A 12} /+$ & $0.71 \pm 0.020$ & 81 \\
\hline imp $\beta 11^{70} /+;$ wit $^{H A 1} /+$ & $0.76 \pm 0.025$ & 86 \\
\hline $\operatorname{imp} \beta 11^{70} /+; n-s y b^{F-33 R} /+$ & $0.85 \pm 0.048$ & 96 \\
\hline imp $\beta 11^{70} /$ UAS-wit; wit ${ }^{\text {HA1 }} /$ elav-GAL4 & $0.92 \pm 0.049$ & 104 \\
\hline $\operatorname{imp} \beta 11^{70} / D f+$ act $R$ neuron rescue & $0.98 \pm 0.040$ & 111 \\
\hline $\operatorname{imp} \beta 11^{70} / D f+$ act $R$ muscle rescue & $0.70 \pm 0.026$ & 80 \\
\hline
\end{tabular}

Bouton numbers per $1000 \mu \mathrm{m}^{2}$ muscle surface area are displayed as mean values and percentages of control. $p$ values and full genotypes are noted in the figure legends.

acute blockade of transmission at third-instar NMJs by means of the use-dependent glutamate receptor antagonist PhTx (Frank et al., 2006). This process fails in $g b b$ and wit mutant larvae, and the $\mathrm{BMP}$ pathway is therefore thought to have a permissive role, although not necessarily to be acutely involved in the upregulation of transmission (Goold and Davis, 2007). To examine this phenomenon, both control and imp $\beta 11$ mutant third-instar larvae were exposed to PhTx for $10 \mathrm{~min}$ and compared with preparations of the same genotypes mock-treated with saline. In both mutants and controls, PhTx decreased mEJP amplitude, whereas EJP amplitude remained unchanged (supplemental Fig. S8, available at www.jneurosci.org as supplemental material). Thus, the mechanisms governing the homeostatic increase in quantal content remained operational in the imp $\beta 11$ larvae, despite the lack of pMAD at these NMJs. Therefore, the local pool of pMAD at the NMJ may not be required acutely for PhTx-induced synaptic homeostasis. Because block of retrograde transport of pMAD has been shown to hinder PhTx-induced homeostasis (Goold and Davis, 2007), nuclear pMAD is most likely responsible for permitting this form of plasticity. Thus, although pMAD is generated in the periphery by BMP signaling, it does not need to remain resident in the terminals for homeostasis to be enabled.

\section{Genetic interaction of $i m p \beta 11$ and the BMP pathway}

The similarity between published BMP pathway mutants and those associated with loss of importin- $\beta 11$ function, as well as the observed reduction in pMAD at imp $\beta 11$ terminals, suggested that importin- $\beta 11$ and the BMP pathway interacted to regulate the development of the synapse. To test this hypothesis, we examined genetic interactions between imp $\beta 11$ and the BMP pathway. Although mutations in imp $\beta 11$ and BMP pathway components each reduce bouton counts, this phenotype is recessive; heterozygotes for these alleles are normal (Figs. 3B, 6A; Table 2). In contrast, larvae made heterozygous for both $i m p \beta 11^{70}$ and either a null allele of $M A D\left(M A D^{10}\right)$ (Sekelsky et al., 1995), a wit null allele $\left(w_{i t}^{A 12}\right)$ (Marqués et al., 2002), or a hypomorphic allele of wit (wit ${ }^{H A 1}$ ) (Aberle et al., 2002) had significantly fewer boutons than either wild-type or each heterozygous alleles alone (Fig. 6A, Table 2 ). These genetic interactions were specific for mutations in the BMP pathway; larvae heterozygous for imp $\beta 11$ and the synaptic transmission mutant neuronal synaptobrevin ( $n-s y b)$ (Deitcher et al., 1998) had normal synaptic arbors. Neuronal expression of
$U A S$-wit was able to reverse the reduction in bouton number in $i m p \beta 11^{70} /+$; wit ${ }^{H A 1} /+$ larvae (Fig. 6A, Table 2). The interaction between importin- $\beta 11$ and BMP components is therefore likely to occur presynaptically and, consistent with a role for importin$\beta 11$ in BMP signaling, resembles the genetic interactions of other heterozygous combinations of mutations in the BMP pathway (McCabe et al., 2004).

Because BMP pathway mutants resemble imp $\beta 11$ mutant alleles, reducing both bouton number and synaptic strength, loss of pMAD could explain these imp $\beta 11$ phenotypes. Restoration of synaptic pMAD in imp $\beta 11$ null larvae would then be predicted to rescue the phenotypes. To this end, we introduced into motor neurons expression of constitutively active receptors with the driver OK6-GAL4 (Aberle et al., 2002) and UAS-tkv ${ }^{a c t}$, sax ${ }^{a c t}$ transgenes (Holley et al., 1996; Hoodless et al., 1996). This expression, but not postsynaptic expression (G14-GAL4), restored pMAD to imp $\beta 11$ mutant terminals, a finding consistent with our conclusion that an absence of MAD cannot account for the loss of pMAD in imp $\beta 11$ mutants (Fig. $6 B-F$ ). Concomitant with pMAD restoration, expression of the activated receptors in motor neurons also rescued the bouton number and EJP phenotypes in imp $\beta 11$ to control levels, although mEJP frequency was not rescued (Fig. 6A, G-J, Table 2). Expression of the activated receptors in muscle (G14-GAL4) did not rescue any of the phenotypes (Fig. 6A, G-J, Table 2). In control larvae, expression of the activated receptors in motor neurons did not change pMAD levels or the electrophysiological profile at the NMJ (Fig. 6C, G-J). Thus, restoring the presynaptic pool of pMAD was sufficient for normal bouton number and EJP amplitude. Moreover, the rescue of these phenotypes in the imp $\beta 11$ mutants cannot be attributed to a simple addition of a $U A S$ - $t k v^{a c t}$, sax $x^{a c t}$-induced increase and an imp $\beta 11$-induced decrease as there is no gain-of-function phenotype, but rather to overcoming a particular deficit in pMAD signaling in the $i m p \beta 11$ genotype.

\section{Reduction of retrograde transport restores imp $\boldsymbol{\beta} 11$ phenotypes}

The levels of pMAD in nerve terminals may also be controlled by the rate at which it, or a component of the pathway, is transported away from the terminal to the nucleus or for degradation. Inhibiting the retrograde motor dynein by expression of p150-Glued blocks retrograde BMP signaling and reduces nuclear pMAD with consequent effects on NMJ development (Eaton et al., 2002; Allan et al., 2003; Marqués et al., 2003; McCabe et al., 2003). The dynein motor may transport pMAD itself or another signaling component required for pMAD production or maintenance. Therefore, we hypothesized that the structural and functional phenotypes of imp $\beta 11$ might also be rescued by inhibiting retrograde transport. Overexpression of dynamitin inhibits transport by destabilizing the dynein motor complex (Duncan and Warrior, 2002; Melkonian et al., 2007), but to a lesser extent than p150Glued expression. When UAS-dynamitin was expressed in motor neurons with the OK6-GAL4 driver in a wild-type background, pMAD immunoreactivity at the NMJ was similar to control levels (Fig. $7 A, B$ ), and nuclear pMAD showed no obvious reduction (Fig. $7 E, F$ ). Bouton number and electrophysiological properties were likewise unchanged (Fig. $7 G-K$ ). When UAS-dynamitin was expressed in the imp $\beta 11$ mutant background, however, the loss of synaptic pMAD was prevented (Fig. $7 C, D$ ). These findings confirmed that the elements of the BMP pathway in the terminal were preserved and at least partially active in the imp $\beta 11$ larvae and suggested that the reduction in synaptic pMAD might arise 

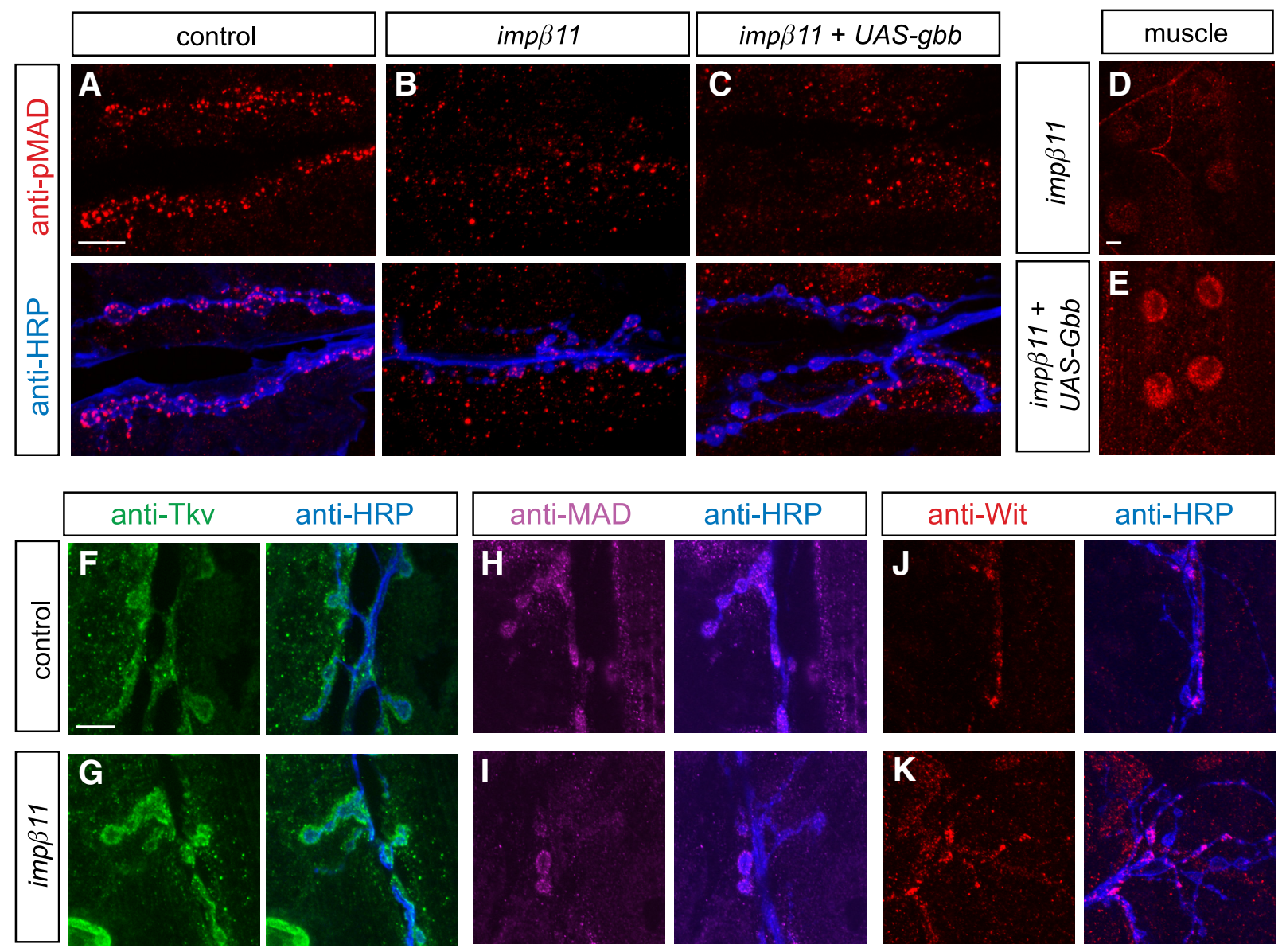

Figure 5. BMP pathway components are present at imp $\beta 11 \mathrm{NMJs.} A-C$, Representative confocal images of third-instar NMJs stained with anti-pMAD (red) and anti-HRP (blue). $\boldsymbol{A}, \boldsymbol{B}$, pMAD was detected at control NMJs $(\boldsymbol{A})$ but was reduced at mutant NMJs $(\boldsymbol{B})$. $\boldsymbol{C}-\boldsymbol{E}$, Overexpression of UAS-gbb in muscle did not restore pMAD to mutant terminals, but did increase pMAD in muscle nuclei $(\boldsymbol{D}, \boldsymbol{E}) . \boldsymbol{F}-\boldsymbol{K}$, Representative confocal images of NMJs from control and imp $\beta 11$ larvae immunostained for anti-HRP (blue) and components of the BMP pathway. $\boldsymbol{F}, \boldsymbol{G}$, In control and mutant NMJs, the BMP receptor Tkv (green) was visible both within and around the boutons. $\boldsymbol{H}-\boldsymbol{K}$, The substrate of BMP receptors, MAD (magenta), and the receptor Wit (red) were present at both mutant and control synapses. Scale bars, $10 \mu \mathrm{m}$. Genotypes are as follows: control ( $y, w ; F R T 42 D)$, imp $\beta 11$ (FRT42D,imp $\beta 11^{70} / D f$ ), imp $\beta 11+$ UAS-gbb (w; FRT42D, imp $\beta 11^{70}$, G14-GAL4/Df; UAS-gbb).

from an excess of retrograde transport away from the terminal. Coincident with pMAD restoration to the mutant terminal, EJP amplitude, mEJP frequency, quantal content, and bouton number were rescued to control values (Fig. $7 G-K$ ). No defect in mEJP amplitude was detected in $i m p \beta 11^{70 / 70}$ larvae (Fig. $7 I$ ). These data indicate that restriction of retrograde transport is sufficient to reverse imp $\beta 11$ phenotypes and suggest that importin$\beta 11$ promotes or stabilizes $\mathrm{pMAD}$ localization to the NMJ, which is in turn required for synapse formation and transmission.

\section{Discussion}

Drosophila mutants have allowed us to examine, in vivo, importin- $\beta 11$ and uncover its synaptic function and relationship with the BMP pathway. Loss of importin- $\beta 11$ causes specific developmental and functional defects: motor neurons develop fewer boutons, and EJP amplitudes are 40\% lower than controls.

Importin- $\beta$ s are structurally diverse; they share a small $\mathrm{N}$-terminal Ran-binding domain but the remaining $\mathrm{C}$ terminus of each is unique and allows for substrate specificity and functional diversity (Mosammaparast and Pemberton, 2004). This diversity extends from interactions with nucleoporins to functions in the cell periphery or neuronal synapses (James et al., 2007; Ting et al., 2007). At these locations, they can begin the process of movement to the nucleus but also have additional functions (Harel and Forbes, 2004; Otis et al., 2006; Perry and Fainzilber, 2009).

The functional specificity of importins is illustrated by the imp $\beta 11$ phenotypes, phenotypes that are not shared by other known importin mutants and arise only in a subset of tissues. For example, mutations in imp13 do not alter NMJ bouton number and enhance rather than decrease neurotransmitter release (Giagtzoglou et al., 2009). Similarly, mutations in imp $\alpha 3$ lead to errors in the axonal projections of photoreceptors, a defect not seen in imp $\beta 11$ (Ting et al., 2007; T. Herman, personal communication). Thus, the loss of this importin does not grossly disrupt all nuclear import, but rather causes a restricted and distinct phenotype. Homozygous null imp $\beta 11$ clones in the eye primordium undergo cell division and differentiation and give rise to outwardly normal photoreceptors with correct axonal projections, but have defects in synaptic transmission. At the NMJ, the phenotype is similarly restricted to a synaptic defect with no apparent compromise in motor neuron viability, differentiation, or axon guidance.

The NMJ defects in imp $\beta 11$ null larvae are partial reductions in bouton number and synaptic strength rather than a complete failure of development or transmission. The electrophysiological defect in photoreceptors is less understood, but sufficient to prevent phototaxis. In addition to these defects, some neuronal sub- 

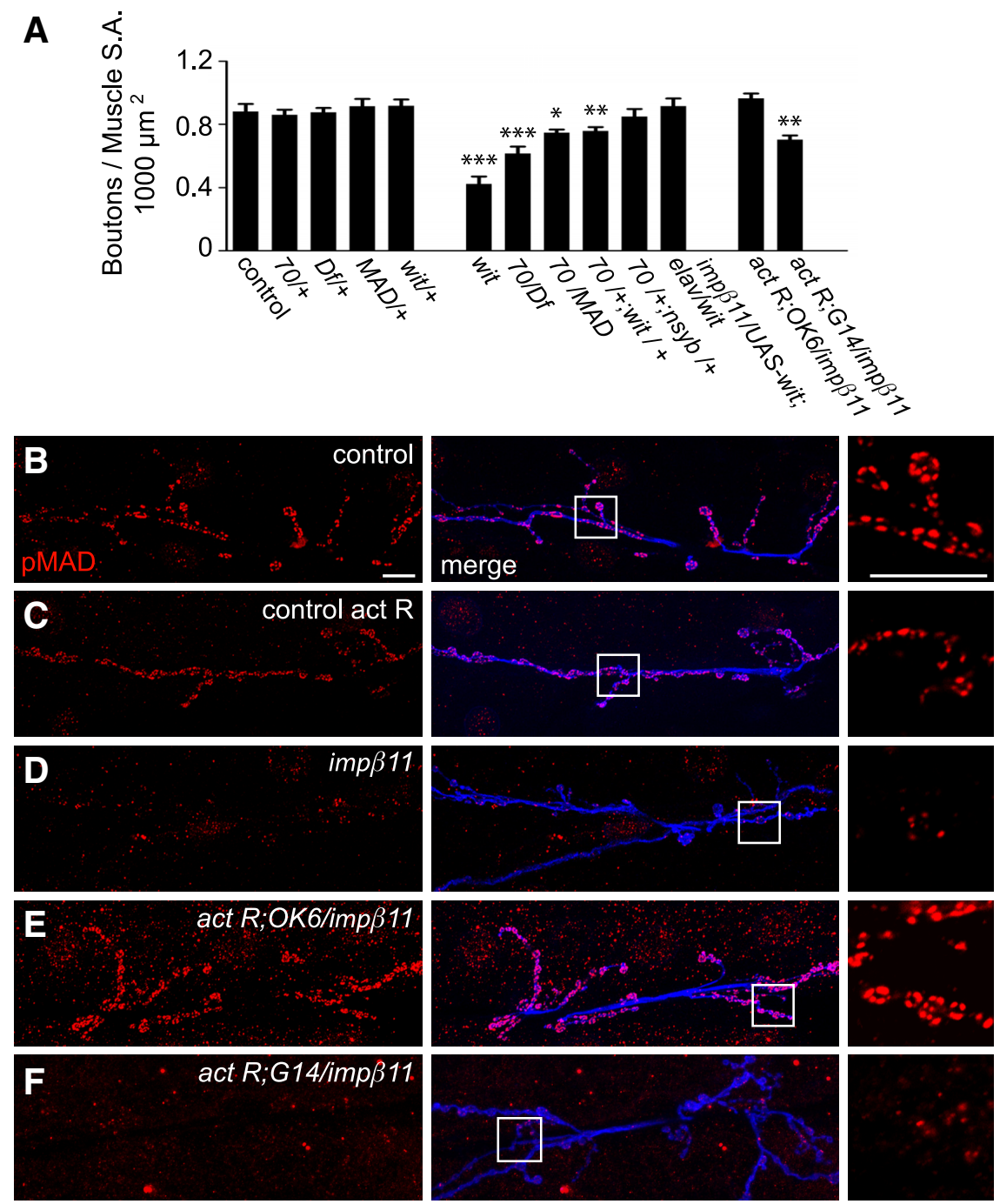

G

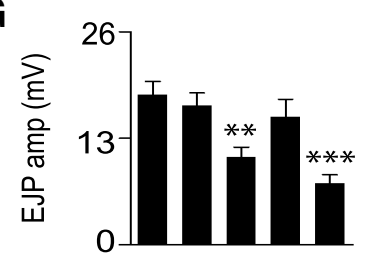

H

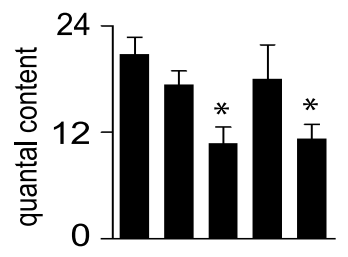

I

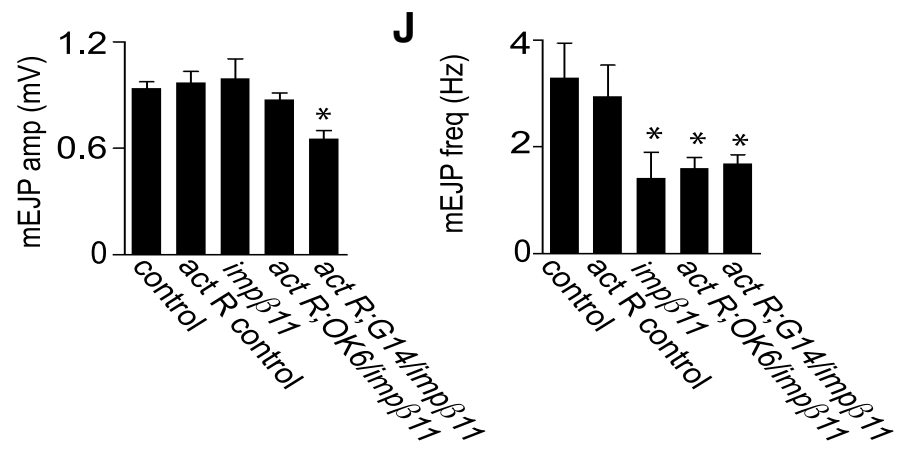

Figure 6. Genetic interaction of imp $\beta 11$ and the BMP pathway. $\boldsymbol{A}, \mathrm{BMP}$ pathway mutants and imp $\beta 11$ interact to form fewer synaptic boutons on muscles 6 and 7. Bouton counts were normal in heterozygotes of $i m p \beta 11^{70}$ or the indicated alleles of BMP pathway mutants but reduced in larvae heterozygous for both $i m p \beta 11^{70}$ and either $M A D^{10}$ or wit ${ }^{H A 1}$, an interaction not seen with $n-s y b^{F-33 R}$. Bouton number was rescued by presynaptic expression of UAS-wit in imp $\beta 11^{70}$; wit ${ }^{H A 1}$ transheterozygotes. In addition, neuronal (but not muscle) expression of constitutively activated BMP receptors (UAS-tkv ${ }^{a c t}$, sax ${ }^{a c t}$ ) restored bouton number in an imp $\beta 11$ mutant background. $\boldsymbol{B}-\boldsymbol{F}$, Representative confocal images of anti-pMAD (red) and anti-HRP (blue) immunoreactivity to illustrate the effects of the constitutively activated BMP receptors on pMAD levels. Scale bars, $10 \mu \mathrm{m}$. In control larvae, the types must be more drastically impaired than motor neurons because neuronal importin- $\beta 11$ is essential for viability. Additional nonneuronal functions are indicated by the failure of oocyte maturation in mutant germline clones, the expression of importin- $\beta 11$ in early embryos, and importin- $\beta 11$ immunoreactivity at the nuclear envelope in muscle. Although importin- $\beta 11$ is expressed in both nerve and muscle, the reduction in bouton number and synaptic strength in imp $\beta 11$ null larvae results primarily from a loss of neuronal importin- $\beta 11$ and can be restored by neuronal expression of importin- $\beta 11$. Other aspects of NMJ development, however, are dependent on importin- $\beta 11$ in muscle (T. J. Mosca and T. L. Schwarz, unpublished observation). Thus, importin- $\beta 11$ functions are both diverse and highly selective.

Of the many factors contributing to NMJ development and function (Keshishian et al., 1996; Collins and DiAntonio, 2007), importin- $\beta 11$ action appears particularly connected to the BMP pathway. The evidence for this connection is fourfold. (1) At imp $\beta 11$ NMJs, pMAD is markedly reduced. Neuronal expression of an imp $\beta 11$ transgene restores pMAD levels in parallel with restoration of bouton number and EJP amplitude. The decrease in pMAD is not part of a widespread loss of synaptic proteins as FasII, Futsch, Syt I, BRP, GluRIIA, GluRIIB, and GluRIIC are not lacking. (2) Mutations in the BMP pathway, including mutations in $M A D$, result in imp $\beta 11$-like phenotypes: fewer boutons and smaller EJPs (Marqués, 2005). (3) Mutations in imp $\beta 11$ interact genetically with mutations in MAD and wit; heterozygous mutations that are recessive

$\leftarrow$

activated receptors driven in motor neurons did not appreciably increase $\operatorname{pMAD}(\boldsymbol{C}$, but in imp $\beta 11$ larvae pMAD levels were restored $(\boldsymbol{E})$. When activated receptors were expressed in imp $\beta 11$ muscle, pMAD staining was not restored $(\boldsymbol{F})$. G-J, EJP amplitude and quantal content, but not mini frequency, were restored to control levels by the activated BMP receptors expressed in motor neurons but not in muscle. Recordings were conducted in $0.6 \mathrm{~mm} \mathrm{Ca}{ }^{2+} \mathrm{HL} 3 ; n \geq 6$ for each genotype. ${ }^{*} p<0.05 ;{ }^{* *} p<0.001 ;{ }^{* * *} p<0.0001$ compared to control. Genotypes are as follows: control ( $y, w$; FRT42D $), 70 /+\left(w ; F R T 42 D, \quad i m p \beta 11^{70} /+\right), \mathrm{MAD} /+$ $\left(M^{\prime} D^{10} /+\right)$, wit/ + $\left(\right.$ wit $\left.^{H A 1} /+\right)$, wit $\left(\right.$ wit $^{A 12} /$ wit $\left.^{B 11}\right), 70 / D f$ (FRT42D, imp $\beta 11^{70} / \mathrm{Df}$ ), 70/MAD (FRT42D, imp $\beta 11^{70}$ ) mad $\left.^{10}\right), 70 /+$; wit $+\left(\right.$ FRT42D, imp $\beta 11^{70} /+;$ wit $\left.^{\text {HA1 }} /+\right)$, $70 /+; \mathrm{n}-\mathrm{syb} /+\left(\right.$ FRT42D, imp $\beta 11^{70} /+; n-s y b^{F-33 R} /+$ ), 70/UAS-wit;elav/wit (FRT42D, imp $\beta 11^{70} /$ UAS-wit; elavGAL4/wit ${ }^{H A 7}$ ), control act R (UAS-tkv ${ }^{a c t}$, UAS-sax ${ }^{a c t}$; OK6GAL4/+), act R;OK6/ imp $\beta 11$ (UAS-tkv ${ }^{a c t}$, UAS-sax ${ }^{a c t}$; FRT42D, imp $\beta 11^{70}$, OK6-GAL4/Df), act R;G14/ imp $\beta 11$ (UAS-tkv ${ }^{a c t}$, UAS-sax ${ }^{a c t}$; FRT42D, imp $\beta 11^{70}$, G14-GAL4/Df). 

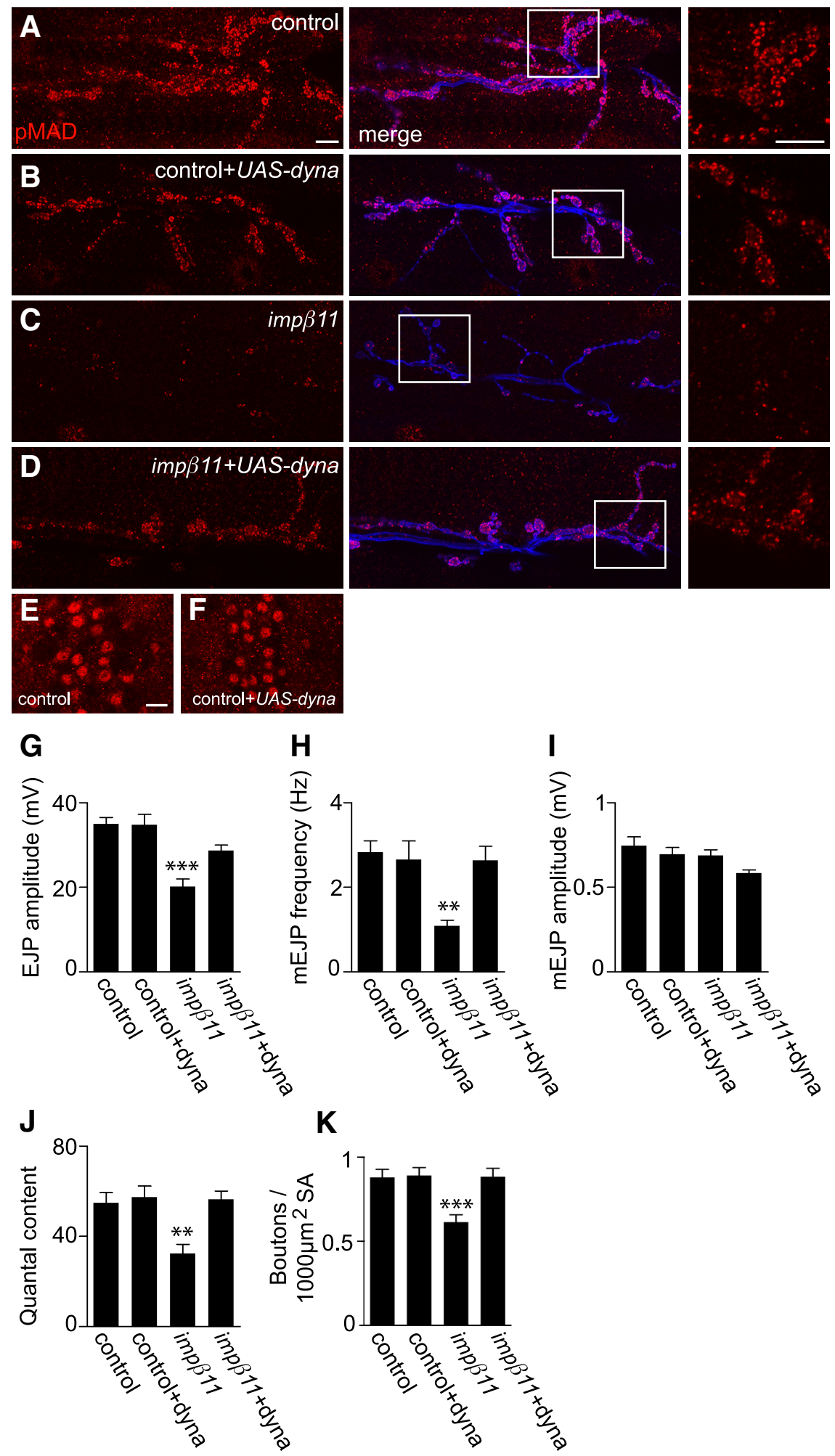

K

Figure 7. Overexpression of dynamitin can reverse imp $\beta 11$ phenotypes. $\boldsymbol{A}-\boldsymbol{D}$, Representative confocal images of NMJs immunostained with anti-pMAD (red) and anti-HRP (blue). Scale bars, $10 \mu \mathrm{m}$. $\boldsymbol{B}-\boldsymbol{F}$, Motor neuron expression of UAS-dynamitin in a control background did not detectably alter pMAD levels at the NMJ or in motor neuron nuclei $(\boldsymbol{B}, \boldsymbol{F})$; when compared with control $(\boldsymbol{A}, \boldsymbol{E})$; however, pMAD was restored to $\operatorname{imp} \beta 11 \mathrm{NMJs}(\boldsymbol{C}, \boldsymbol{D})$. G-J, Expression of dynamitin in a control background did not alter EJP amplitude, mEJP frequency, or quantal content, but reversed the reduction in those parameters in imp $\beta 11$ mutants (EJP amplitude: control, $35.0 \pm 1.56 \mathrm{mV}$; control+dyna, $34.8 \pm 2.45 \mathrm{mV}$; imp $\beta 11,20.1 \pm 1.84 \mathrm{mV}$; imp $\beta 11+$ dyna, $28.7 \pm 1.30 \mathrm{mV}$ mEJP frequency: control, $2.83 \pm 0.274 \mathrm{~Hz}$; control+dyna, $2.66 \pm 0.441 \mathrm{~Hz}$; imp $\beta 11,1.08 \pm 0.142 \mathrm{~Hz} ; i m p \beta 11+$ dyna, $2.64 \pm$ $0.331 \mathrm{~Hz}$; quantal content: control, $54.8 \pm 4.55$; control+dyna, $57.4 \pm 4.90 ; i m p \beta 11,32.5 \pm 4.10 ; i m p \beta 11+$ dyna, $56.3 \pm 3.86)$. I, mEJP amplitude was normal in all genotypes. $\boldsymbol{K}$, Similarly, dynamitin overexpression in a control background did not alter bouton number but in imp $\beta 11$ restored bouton number to control values. ${ }^{* *} p<0.001 ;{ }^{* * *} p<0.0001$ compared to independently interact to reduce bouton number when combined. These genetic interactions with imp $\beta 11$ are comparable to those between established BMP-pathway members (McCabe et al., 2004). (4) Restoration of synaptic pMAD levels in imp $\beta 11$ mutants either by constitutively activated BMP receptors or impaired retrograde transport also rescues the bouton and EJP phenotypes.

These data suggest that, under normal conditions, importin- $\beta 11$ permits a synaptic pool of pMAD to exist and that this pool of presynaptic pMAD is required for normal NMJ structure and function. Although SMADs chiefly act as transcription factors, there is growing precedent for alternative posttranscriptional functions (Davis et al., 2008; Hoover and Kubalak, 2008; Tang et al., 2008). A local, synaptic function of $\mathrm{pMAD}$ presently provides the simplest explanation of the correlation of the imp $\beta 11$ null phenotypes and local pMAD levels. The mechanism underlying the reduction in synaptic pMAD, however, remains unknown. We have been unable to detect by immunoprecipitation a direct interaction between importin- $\beta 11$ and several members of the BMP pathway (data not shown). Moreover, the essential elements of the pathway, Wit, Tkv, and MAD itself, remain in the mutant terminals, and overexpression of GFP-MAD neither restores pMAD levels nor alleviates the imp $\beta 11$ phenotype. Therefore, a failure to transcribe or traffic these proteins cannot account for the loss of pMAD. In contrast, overactivation of the receptors by constitutively activated tk $v$ and sax overcomes the imp $\beta 11$ phenotype. A standard genetic explanation of this rescue by activated receptors would be that the disruption of the pathway is upstream of the receptor, in this case loss of Gbb secretion by the muscle. This is unlikely to explain the imp $\beta 11$ phenotype, however, because importin- $\beta 11$ is required in the nervous system, not muscle, for maintaining normal pMAD levels. Furthermore, overexpression of Gbb does not suppress the loss of $\mathrm{pMAD}$ at imp $\beta 11$ synapses. Thus, fundamentals of the BMP pathway, the receptors, and their ligand and substrate, are intact at the imp $\beta 11$ NMJ. Indeed, the continued function-

$\leftarrow$

control; $n \geq 8$ animals for each genotype. Genotypes are as follows: control $(y, w ;$ FRT42D), control+dyna (UASdynamitin/OK6-GAL4), imp $\beta 11$ (w;FRT42D, imp $\beta 11^{70 / 70}$ ), imp $\beta 11+$ dyna (UAS-dynamitin, FRT42D, imp $\beta 11^{70} /$ FRT42D, imp $\beta 11^{70}$, OK6-GAL4). 
ing of the pathway is suggested by the presence of nuclear pMAD in the motor neurons and by the occurrence of homeostatic compensation to $\mathrm{PhTx}$ at the synapse, a process that fails in mutations of the BMP pathway (Goold and Davis, 2007). Instead, the loss of synaptic pMAD in imp $\beta 11$ may likely arise from a modulation of this pathway that causes pMAD to be lost locally from the terminals while keeping intact the pathway for pMAD production and nuclear localization.

Excess retrograde transport of pMAD from the NMJ might plausibly explain pMAD loss in imp $\beta 11$. Importin- $\beta 11$ might inhibit, either directly or indirectly, retrograde transport of pMAD from terminals, and thereby retain a pool of synaptic pMAD for local functions. This hypothesis is consistent with an intact BMP pathway, persistent nuclear pMAD in motor neurons, and selective loss of synaptic pMAD. Synaptic receptors are needed for nuclear $\mathrm{pMAD}$ in a pathway that depends on retrograde transport (Marqués, 2005). Although we do not see increased nuclear pMAD in mutant motor neurons, increased transport out of the terminals need not cause a detectable increase in the nuclear pool. pMAD-dependent transcriptional regulation likely promotes synapse development, probably as a permissive signal for NMJ growth (Collins and DiAntonio, 2007; Goold and Davis, 2007). Synaptic pMAD may instead maintain or remodel the NMJ more subtly. This difference can explain why mutations in wit, which reduce pMAD in both terminals and nuclei, cause stronger synaptic defects than imp $\beta 11$ (Aberle et al., 2002; Marqués et al., 2002). Other possible explanations of the pMAD phenotype include a role for importin- $\beta 11$ in protecting the synaptic pool from dephosphorylation or degradation, or a modulatory effect of the importin on the basal activation of the BMP receptors. In addition, we cannot rule out subtle alterations in nuclear pMAD caused by potential redundancy of importin$\beta 11$ with other importins (Xu et al., 2007) or effects of maternally contributed importin- $\beta 11$.

A defect in nuclear import might indirectly, perhaps through transcriptional changes, control synaptic pMAD levels. Such a mechanism would be consistent with importin- $\beta 11$ localization at neuronal nuclear pores and with the canonical function of importins. However, because importin- $\beta 11$-eGFP localizes to the NMJ and other importins occur at synapses (Thompson et al., 2004; Ting et al., 2007), importin- $\beta 11$ might instead act synaptically to modulate pMAD. Whether acting locally, at the nuclear pore, or both, our data reveal a potentially novel function for an importin in regulating BMP signaling. Because many other factors and pathways interact with BMP signaling (von Bubnoff and Cho, 2001; Keshishian and Kim, 2004; Collins and DiAntonio, 2007), there are many potential targets for further investigation of importin- $\beta 11$ mechanisms.

Although importins have primarily been examined at the nuclear pore, they also undergo long-distance translocations in the cytoplasm that can be important for signal transduction (Guillemain et al., 2002; James et al., 2007). These functions are of particular interest in the nervous system and include synapse-to-nucleus shuttling where they are implicated in synaptic plasticity, axonal regeneration, and axonal targeting (Otis et al., 2006; Ting et al., 2007; Perry and Fainzilber, 2009). With the characterization of Drosophila imp $\beta 11$, the regulation of synaptic development and strength can be added to the repertoire of importin-dependent functions, whereas the phenotype of the mutations serves to highlight the functional selectivity of an individual importin.

\section{References}

Aberle H, Haghighi AP, Fetter RD, McCabe BD, Magalhaes TR, Goodman CS (2002) Wishful thinking encodes a BMP type II receptor that regulates synaptic growth in Drosophila. Neuron 33:545-558.

Allan DW, St Pierre SE, Miguel-Aliaga I, Thor S (2003) Specification of neuropeptide cell identity by the integration of retrograde BMP signaling and a combinatorial transcription factor code. Cell 113:73-86.

Anderson KV, Lengyel JA (1979) Rates of synthesis of major classes of RNA in Drosophila embryos. Dev Biol 70:217-231.

Boll W, Noll M (2002) The Drosophila Pox neuro gene: control of male courtship behavior and fertility as revealed by a complete dissection of all enhancers. Development 129:5667-5681.

Brand AH, Perrimon N (1993) Targeted gene expression as a means of altering cell fates and generating dominant phenotypes. Development 118:401-415.

Caesar S, Greiner M, Schlenstedt G (2006) Kap120 functions as a nuclear import receptor for ribosome assembly factor Rpf1 in yeast. Mol Cell Biol 26:3170-3180.

Chan CC, Zhang S, Rousset R, Wharton KA (2008) Drosophila Naked cuticle (Nkd) engages the nuclear import adaptor Importin-alpha3 to antagonize Wnt/beta-catenin signaling. Dev Biol 318:17-28.

Chou TB, Perrimon N (1992) Use of a yeast site-specific recombinase to produce female germline chimeras in Drosophila. Genetics 131:643-653.

Chou TB, Perrimon N (1996) The autosomal FLP-DFS technique for generating germline mosaics in Drosophila melanogaster. Genetics 144:16731679.

Collins CA, DiAntonio A (2007) Synaptic development: insights from Drosophila. Curr Opin Neurobiol 17:35-42.

Collins CA, Wairkar YP, Johnson SL, DiAntonio A (2006) Highwire restrains synaptic growth by attenuating a MAP kinase signal. Neuron 51:57-69.

Coombe PE (1986) The large monopolar cells L1 and L2 are responsible for ERG transients in Drosophila. J Comp Physiol A Neuroethol Sens Neural Behav Physiol 159:655-665.

Davis BN, Hilyard AC, Lagna G, Hata A (2008) SMAD proteins control DROSHA-mediated microRNA maturation. Nature 454:56-61.

Deitcher DL, Ueda A, Stewart BA, Burgess RW, Kidokoro Y, Schwarz TL (1998) Distinct requirements for evoked and spontaneous release of neurotransmitter are revealed by mutations in the Drosophila gene neuronalsynaptobrevin. J Neurosci 18:2028-2039.

DiAntonio A, Parfitt KD, Schwarz TL (1993) Synaptic transmission persists in synaptotagmin mutants of Drosophila. Cell 73:1281-1290.

DiAntonio A, Petersen SA, Heckmann M, Goodman CS (1999) Glutamate receptor expression regulates quantal size and quantal content at the Drosophila neuromuscular junction. J Neurosci 19:3023-3032.

Dickman D, Horne JA, Meinertzhagen IA, Schwarz TL (2005) A slowed classical pathway rather than kiss-and-run mediates endocytosis at synapses lacking synaptojanin and endophilin. Cell 123:521-533.

Dickman D, Kurshan P, Schwarz TL (2008) Mutations in a Drosophila $\alpha_{2}$ delta voltage-gated calcium channel subunit reveal a crucial synaptic function. J Neurosci 28:31-38.

Dudu V, Bittig T, Entchev E, Kicheva A, Julicher F, Gonzalez-Gaitan M (2006) Postsynaptic mad signaling at the Drosophila neuromuscular junction. Curr Biol 16:625-635.

Duncan JE, Warrior R (2002) The cytoplasmic dynein and kinesin motors have interdependent roles in patterning the Drosophila oocyte. Curr Biol 12:1982-1991.

Eaton BA, Fetter RD, Davis GW (2002) Dynactin is necessary for synapse stabilization. Neuron 34:729-741.

Fabian-Fine R, Verstreken P, Hiesinger PR, Horne JA, Kostyleva R, Zhou Y, Bellen HJ, Meinertzhagen IA (2003) Endophilin promotes a late step in endocytosis at glial invaginations in Drosophila photoreceptor terminals. J Neurosci 23:10732-10744.

Finlay DR, Newmeyer DD, Price TM, Forbes DJ (1987) Inhibition of in vitro nuclear transport by a lectin that binds to nuclear pores. J Cell Biol 104:189-200.

Frank CA, Kennedy MJ, Goold CP, Marek KW, Davis GW (2006) Mechanisms underlying the rapid induction and sustained expression of synaptic homeostasis. Neuron 52:663-677.

Fried H, Kutay U (2003) Nucleocytoplasmic transport: taking an inventory. Cell Mol Life Sci 60:1659-1688.

Giagtzoglou N, Lin YQ, Haueter C, Bellen HJ (2009) Importin 13 regulates 
neurotransmitter release at the Drosophila neuromuscular junction. J Neurosci 29:5628-5639.

Goodman CS, Shatz CJ (1993) Developmental mechanisms that generate precise patterns of neuronal connectivity. Cell [Suppl] 72:77-98.

Goold CP, Davis GW (2007) The BMP ligand Gbb gates the expression of synaptic homeostasis independent of synaptic growth control. Neuron 56:109-123.

Görlich D, Kutay U (1999) Transport between the cell nucleus and the cytoplasm. Annu Rev Cell Dev Biol 15:607-660.

Görlich D, Kostka S, Kraft R, Dingwall C, Laskey RA, Hartmann E, Prehn S (1995) Two different subunits of importin cooperate to recognize nuclear localization signals and bind them to the nuclear envelope. Curr Biol 5:383-392.

Greer PL, Greenberg ME (2008) From synapse to nucleus: calciumdependent gene transcription in the control of synapse development and function. Neuron 59:846-860.

Guillemain G, Muñoz-Alonso MJ, Cassany A, Loizeau M, Faussat AM, Burnol AF, Leturque A (2002) Karyopherin alpha2: a control step of glucose-sensitive gene expression in hepatic cells. Biochem J 364:201-209.

Haerry TE, Khalsa O, O’Connor MB, Wharton KA (1998) Synergistic signaling by two BMP ligands through the SAX and TKV receptors controls wing growth and patterning in Drosophila. Development 125:3977-3987.

Hanz S, Perlson E, Willis D, Zheng JQ, Massarwa R, Huerta JJ, Koltzenburg M, Kohler M, van-Minnen J, Twiss JL, Fainzilber M (2003) Axoplasmic importins enable retrograde injury signaling in lesioned nerve. Neuron 40:1095-1104.

Harel A, Forbes DJ (2004) Importin beta: conducting a much larger cellular symphony. Mol Cell 16:319-330.

Harlow E, Lane D (1988) Antibodies: a laboratory manual. Cold Spring Harbor, NY: Cold Spring Harbor Laboratory.

Hazelett DJ, Bourouis M, Walldorf U, Treisman JE (1998) decapentaplegic and wingless are regulated by eyes absent and eyegone and interact to direct the pattern of retinal differentiation in the eye disc. Development 125:3741-3751.

Heisenberg M (1971) Separation of receptor and lamina potentials in the electroretinogram of normal and mutant Drosophila. J Exp Biol 55:85-100.

Hiesinger PR, Zhai RG, Zhou Y, Koh TW, Mehta SQ, Schulze KL, Cao Y, Verstreken P, Clandinin TR, Fischbach KF, Meinertzhagen IA, Bellen HJ (2006) Activity-independent prespecification of synaptic partners in the visual map of Drosophila. Curr Biol 16:1835-1843.

Hoang B, Chiba A (2001) Single-cell analysis of Drosophila larval neuromuscular synapses. Dev Biol 229:55-70.

Hofbauer A, Ebel T, Waltenspiel B, Oswald P, Chen YC, Halder P, Biskup S, Lewandrowski U, Winkler C, Sickmann A, Buchner S, Buchner E (2009) The Wuerzburg hybridoma library against Drosophila brain. J Neurogenet 23:78-91.

Holley SA, Neul JL, Attisano L, Wrana JL, Sasai Y, O’Connor MB, De Robertis EM, Ferguson EL (1996) The Xenopus dorsalizing factor noggin ventralizes Drosophila embryos by preventing DPP from activating its receptor. Cell 86:607-617.

Hoodless PA, Haerry T, Abdollah S, Stapleton M, O'Connor MB, Attisano L, Wrana JL (1996) MADR1, a MAD-related protein that functions in BMP2 signaling pathways. Cell 85:489-500.

Hoover LL, Kubalak SW (2008) Holding their own: the noncanonical roles of Smad proteins. Sci Signal 1:pe48.

Hummel T, Krukkert K, Roos J, Davis G, Klämbt C (2000) Drosophila Fut$\mathrm{sch} / 22 \mathrm{C} 10$ is a MAP1B-like protein required for dendritic and axonal development. Neuron 26:357-370.

Jakel S, Mingot JM, Schwarzmaier P, Hartmann E, Gorlich D (2002) Importins fulfill a dual function as nuclear import receptors and cytoplasmic chaperones for exposed basic domains. EMBO J 21:377-386.

James BP, Bunch TA, Krishnamoorthy S, Perkins LA, Brower DL (2007) Nuclear localization of the ERK MAP kinase mediated by Drosophila alphaPS2betaPS integrin and importin-7. Mol Biol Cell 18:4190-4199.

Johansen J, Halpern ME, Johansen KM, Keshishian H (1989) Stereotypic morphology of glutamatergic synapses on identified muscle cells of Drosophila larvae. J Neurosci 9:710-725.

Kelly LE (1983) The regulation of phosphorylation of a specific protein in synaptosomal fractions from Drosophila heads: the effects of light and two visual mutants. Cell Mol Neurobiol 3:127-141.

Keshishian H, Kim YS (2004) Orchestrating development and function: ret- rograde BMP signaling in the Drosophila nervous system. Trends Neurosci 27:143-147.

Keshishian H, Broadie K, Chiba A, Bate M (1996) The drosophila neuromuscular junction: a model system for studying synaptic development and function. Annu Rev Neurosci 19:545-575.

Kittel RJ, Wichmann C, Rasse TM, Fouquet W, Schmidt M, Schmid A, Wagh DA, Pawlu C, Kellner RR, Willig KI, Hell SW, Buchner E, Heckmann M, Sigrist SJ (2006) Bruchpilot promotes active zone assembly, $\mathrm{Ca}^{2+}$ channel clustering, and vesicle release. Science 312:1051-1054.

Laemmli UK (1970) Cleavage of structural proteins during the assembly of the head of bacteriophage T4. Nature 227:680-685.

Lai KO, Zhao Y, Ch'ng TH, Martin KC (2008) Importin-mediated retrograde transport of CREB2 from distal processes to the nucleus in neurons. Proc Natl Acad Sci U S A 105:17175-17180.

Lee T, Luo L (1999) Mosaic analysis with a repressible cell marker for studies of gene function in neuronal morphogenesis. Neuron 22:451-461.

Li J, Li WX (2006) A novel function of Drosophila eIF4A as a negative regulator of Dpp/BMP signalling that mediates SMAD degradation. Nat Cell Biol 8:1407-1414.

Lnenicka GA, Keshishian H (2000) Identified motor terminals in Drosophila larvae show distinct differences in morphology and physiology. J Neurobiol 43:186-197.

Luo L, Liao Y, Jan LY, Jan YN (1994) Distinct morphogenetic functions of similar small GTPases: Drosophila Dracl is involved in axonal outgrowth and myoblast fusion. Genes Dev 8:1787-1802.

Mackler JM, Drummond JA, Loewen CA, Robinson IM, Reist NE (2002) The $\mathrm{C}(2) \mathrm{B} \mathrm{Ca}(2+)$-binding motif of synaptotagmin is required for synaptic transmission in vivo. Nature 418:340-344.

Marqués G (2005) Morphogens and synaptogenesis in Drosophila. J Neurobiol 64:417-434.

Marqués G, Bao H, Haerry TE, Shimell MJ, Duchek P, Zhang B, O’Connor MB (2002) The Drosophila BMP type II receptor Wishful Thinking regulates neuromuscular synapse morphology and function. Neuron 33:529-543.

Marqués G, Haerry TE, Crotty ML, Xue M, Zhang B, O’Connor MB (2003) Retrograde Gbb signaling through the Bmp type 2 receptor wishful thinking regulates systemic FMRFa expression in Drosophila. Development 130:5457-5470

Marrus SB, DiAntonio A (2004) Preferential localization of glutamate receptors opposite sites of high presynaptic release. Curr Biol 14:924-931.

Marrus SB, Portman SL, Allen MJ, Moffat KG, DiAntonio A (2004) Differential localization of glutamate receptor subunits at the Drosophila neuromuscular junction. J Neurosci 24:1406-1415.

Martin AR (1955) A further study of the statistical composition on the endplate potential. J Physiol 130:114-122.

McCabe BD, Marqués G, Haghighi AP, Fetter RD, Crotty ML, Haerry TE, Goodman CS, O'Connor MB (2003) The BMP homolog Gbb provides a retrograde signal that regulates synaptic growth at the Drosophila neuromuscular junction. Neuron 39:241-254.

McCabe BD, Hom S, Aberle H, Fetter RD, Marqués G, Haerry TE, Wan H, O'Connor MB, Goodman CS, Haghighi AP (2004) Highwire regulates presynaptic BMP signaling essential for synaptic growth. Neuron 41:891-905.

Meinertzhagen IA (1996) Ultrastructure and quantification of synapses in the insect nervous system. J Neurosci Methods 69:59-73.

Melkonian KA, Maier KC, Godfrey JE, Rodgers M, Schroer TA (2007) Mechanism of dynamitin-mediated disruption of dynactin. J Biol Chem 282:19355-19364.

Merino C, Penney J, Gonzalez M, Tsurudome K, Moujahidine M, O’Connor MB, Verheyen EM, Haghighi P (2009) Nemo kinase interacts with Mad to coordinate synaptic growth at the Drosophila neuromuscular junction. J Cell Biol 185:713-725.

Moroianu J, Hijikata M, Blobel G, Radu A (1995) Mammalian karyopherin alpha 1 beta and alpha 2 beta heterodimers: alpha 1 or alpha 2 subunit binds nuclear localization signal and beta subunit interacts with peptide repeat-containing nucleoporins. Proc Natl Acad Sci U S A 92:6532-6536.

Mosammaparast N, Pemberton L (2004) Karyopherins: from nucleartransport mediators to nuclear-function regulators. Trends Cell Biol 14:547-556.

Murthy M, Ranjan R, Denef N, Higashi ME, Schupbach T, Schwarz TL (2005) Sec6 mutations and the Drosophila exocyst complex. J Cell Sci 118:1139-1150. 
Nellen D, Burke R, Struhl G, Basler K (1996) Direct and long-range action of a DPP morphogen gradient. Cell 85:357-368.

O'Connor-Giles KM, Ho LL, Ganetzky B (2008) Nervous wreck interacts with thickveins and the endocytic machinery to attenuate retrograde BMP signaling during synaptic growth. Neuron 58:507-518.

Otis KO, Thompson KR, Martin KC (2006) Importin-mediated nuclear transport in neurons. Curr Opin Neurobiol 16:329-335.

Pack-Chung E, Kurshan P, Dickman D, Schwarz TL (2007) A Drosophila kinesin required for synaptic bouton formation and synaptic vesicle transport. Nat Neurosci 10:980-989.

Paine PL, Moore LC, Horowitz SB (1975) Nuclear envelope permeability. Nature 254:109-114.

Perlson E, Hanz S, Ben-Yaakov K, Segal-Ruder Y, Seger R, Fainzilber M (2005) Vimentin-dependent spatial translocation of an activated MAP kinase in injured nerve. Neuron 45:715-726.

Perry RB, Fainzilber M (2009) Nuclear transport factors in neuronal function. Semin Cell Dev Biol 20:600-606.

Persson U, Izumi H, Souchelnytskyi S, Itoh S, Grimsby S, Engstrom U, Heldin CH, Funa K, ten Dijke P (1998) The L45 loop in type I receptors for TGF-beta family members is a critical determinant in specifying Smad isoform activation. FEBS Lett 434:83-87.

Peters R (1983) Nuclear envelope permeability measured by fluorescence microphotolysis of single liver cell nuclei. J Biol Chem 258:11427-11429.

Petersen SA, Fetter RD, Noordermeer JN, Goodman CS, DiAntonio A (1997) Genetic analysis of glutamate receptors in Drosophila reveals a retrograde signal regulating presynaptic transmitter release. Neuron 19:1237-1248.

Plafker SM, Macara IG (2000) Importin-11, a nuclear import receptor for the ubiquitin-conjugating enzyme, UbcM2. EMBO J 19:5502-5513.

Plafker SM, Macara IG (2002) Ribosomal protein L12 uses a distinct nuclear import pathway mediated by importin 11. Mol Cell Biol 22:1266-1275.

Plafker SM, Plafker KS, Weissman AM, Macara IG (2004) Ubiquitin charging of human class III ubiquitin-conjugating enzymes triggers their nuclear import. J Cell Biol 167:649-659.

Rexach M, Blobel G (1995) Protein import into nuclei: association and dissociation reactions involving transport substrate, transport factors, and nucleoporins. Cell 83:683-692.

Riemer D, Stuurman N, Berrios M, Hunter C, Fisher PA, Weber K (1995) Expression of Drosophila lamin C is developmentally regulated: analogies with vertebrate A-type lamins. J Cell Sci 108:3189-3198.

Roos J, Hummel T, Ng N, Klämbt C, Davis GW (2000) Drosophila Futsch regulates synaptic microtubule organization and is necessary for synaptic growth. Neuron 26:371-382.

Rubin GM, Spradling AC (1982) Genetic transformation of Drosophila with transposable element vectors. Science 218:348-353.

Schuster CM, Davis GW, Fetter RD, Goodman CS (1996) Genetic dissection of structural and functional components of synaptic plasticity. I. Fasciclin II controls synaptic stabilization and growth. Neuron 17: 641-654.

Schwarz TL (2006) Transmitter release at the neuromuscular junction. Int Rev Neurobiol 75:105-144.

Sekelsky JJ, Newfeld SJ, Raftery LA, Chartoff EH, Gelbart WM (1995) Genetic characterization and cloning of mothers against dpp, a gene re- quired for decapentaplegic function in Drosophila melanogaster. Genetics 139:1347-1358.

Speese SD, Budnik V (2007) Wnts: up-and-coming at the synapse. Trends Neurosci 30:268-275.

Stewart BA, Atwood HL, Renger JJ, Wang J, Wu CF (1994) Improved stability of Drosophila larval neuromuscular preparations in haemolymphlike physiological solutions. J Comp Physiol A Neuroethol Sens Neural Behav Physiol 175:179-191.

Stowers RS, Schwarz TL (1999) A genetic method for generating Drosophila eyes composed exclusively of mitotic clones of a single genotype. Genetics 152:1631-1639.

Stowers RS, Megeath LJ, Gorska-Andrzejak J, Meinertzhagen IA, Schwarz TL (2002) Axonal transport of mitochondria to synapses depends on milton, a novel Drosophila protein. Neuron 36:1063-1077.

Tang Y, Liu Z, Zhao L, Clemens TL, Cao X (2008) Smad7 stabilizes betacatenin binding to E-cadherin complex and promotes cell-cell adhesion. J Biol Chem 283:23956-23963.

Thompson KR, Otis KO, Chen DY, Zhao Y, O’Dell TJ, Martin KC (2004) Synapse to nucleus signaling during long-term synaptic plasticity; a role for the classical active nuclear import pathway. Neuron 44:997-1009.

Ting CY, Herman T, Yonekura S, Gao S, Wang J, Serpe M, O'Connor MB, Zipursky SL, Lee CH (2007) Tiling of r7 axons in the Drosophila visual system is mediated both by transduction of an activin signal to the nucleus and by mutual repulsion. Neuron 56:793-806.

von Bubnoff A, Cho KW (2001) Intracellular BMP signaling regulation in vertebrates: pathway or network? Dev Biol 239:1-14.

Vrailas AD, Marenda DR, Cook SE, Powers MA, Lorenzen JA, Perkins LA, Moses K (2006) smoothened and thickveins regulate Moleskin/Importin 7-mediated MAP kinase signaling in the developing Drosophila eye. Development 133:1485-1494.

Wagh D, Rasse TM, Asan E, Hofbauer A, Schwenkert I, Durrbeck H, Buchner S, Dabauvalle M, Schmidt M, Qin G (2006) Bruchpilot, a protein with homology to ELKS/CAST, is required for structural integrity and function of synaptic active zones in Drosophila. Neuron 49:833-844.

Wang X, Shaw W, Tsang H, Reid E, O'Kane C (2007) Drosophila spichthyin inhibits BMP signaling and regulates synaptic growth and axonal microtubules. Nat Neurosci 10:177-185.

Wharton KA, Cook JM, Torres-Schumann S, de Castro K, Borod E, Phillips DA (1999) Genetic analysis of the bone morphogenetic protein-related gene, gbb, identifies multiple requirements during Drosophila development. Genetics 152:629-640.

Wodarz A, Hinz U, Engelbert M, Knust E (1995) Expression of crumbs confers apical character on plasma membrane domains of ectodermal epithelia of Drosophila. Cell 82:67-76.

Xu L, Yao X, Chen X, Lu P, Zhang B, Ip YT (2007) Msk is required for nuclear import of TGF-\{beta\}/BMP-activated Smads. J Cell Biol 178: 981-994.

Xu T, Rubin GM (1993) Analysis of genetic mosaics in developing and adult Drosophila tissues. Development 117:1223-1237.

Zhu H, Kavsak P, Abdollah S, Wrana JL, Thomsen GH (1999) A SMAD ubiquitin ligase targets the BMP pathway and affects embryonic pattern formation. Nature 400:687-693. 